Int. J. Contemp. Math. Sciences, Vol. 1, 2006, no. 14, 679 - 696

\title{
On rank 3 residually connected geometries for $M_{23}$
}

\author{
Nayil Kilic \\ Harran University, Arts and Science Faculty \\ Department of Mathematics \\ 63300, Sanliurfa, Turkey \\ nayilkilic_61@yahoo.co.uk
}

\begin{abstract}
In this paper we determine all rank 3 residually connected geometries for the Mathieu group $M_{23}$ for which object stabilizers are maximal subgroups.
\end{abstract}

Mathematics Subject Classification: 20D08, 51E10, 05C25

Keywords: Mathieu groups, Steiner system, group geometries

\section{Introduction and Notation}

We begin by reviewing geometries and some standard notation. Let $I, \Gamma$ be sets, where $I$ is a finite and let $t$ be map from $\Gamma$ to $I$. Then the triple $(\Gamma, *, t)$, where $*$ is a symmetric incidence relation on $\Gamma$, is a geometry provided that whenever $x * y(x, y \in \Gamma)$ then $t(x) \neq t(y)$. It is usual we will do it here, to write $\Gamma$ instead of $(\Gamma, *, t)$ and to say $\Gamma$ is a geometry. The map $t: \Gamma \rightarrow I$ is called the type map and we say $x \in \Gamma$ has type $i$ if $t(x)=i$. Also for $x, y \in \Gamma$ if $x * y$, then we will say $x$ and $y$ are incident. The rank of the geometry is the cardinality of $t(\Gamma)$. For $i \in I, \Gamma_{i}=\{x \in \Gamma \mid t(x)=i\}$; so $\Gamma_{i}$ consist of all elements of $\Gamma$ which have type $i$. Suppose $\Gamma$ is a geometry, for $x \in \Gamma$, the residue of $x$ is $\Gamma_{x}=\{y \in \Gamma \mid x * y\}$. The notation of the residue is important in the theory of geometries note that $\left(\Gamma_{x}, * \mid \Gamma_{x}, t\right)$ is a geometry in its own right (where $* \mid \Gamma_{x}$ is the restriction of $*$ to $\Gamma_{x}$ ). Also we note that for every $y \in \Gamma_{x}$, $t(x) \neq t(y)$. A flag $F$ of $\Gamma$ is a subset of $\Gamma$ which, for all $x, y \in F, x \neq y, x * y$. Let $\Gamma$ be a geometry and $F$ a flag of $\Gamma$. The type of $F$ is the subset $t(F)$ of $I$ and the rank (respectively corank) of $F$ is the cardinality of $t(F)$ (respectively $I \backslash t(F)$ ). A chamber of $\Gamma$ is flag of rank $I$. All geometries we consider are assumed to contain at least one flag of rank $|I|$. The automorphism group of $\Gamma, A u t \Gamma$, consist of all permutations of $\Gamma$ which preserve the sets $\Gamma_{i}$ and the incidence relation * . Let $G$ be a subgroup of $A u t \Gamma$. We call $\Gamma$ a flag transitive 
geometry for $G$ if for any two flags $F_{1}$ and $F_{2}$ of $\Gamma$ having the same type, there exists $g \in G$ such that $F_{1}{ }^{g}=F_{2}$.

A geometry $\Gamma$ is called residually connected if for all flags $F$ of $\Gamma$ of corank 2 the incidence graph of $\Gamma_{F}$ is connected. Now suppose that $\Gamma$ is a flag transitive geometry for the group $G$. As is well-known we may view $\Gamma$ in terms of certain cosets of $G$. This is the approach we shall follow here. For each $i \in I$ choose an $x_{i} \in \Gamma_{i}$ and set $G_{i}=\operatorname{Stab}_{G}\left(x_{i}\right)$. Let $\mathcal{F}=\left\{G_{i}: i \in I\right\}$. We now define a geometry $\Gamma(G, \mathcal{F})$ where the objects of type $i$ in $\Gamma(G, \mathcal{F})$ are the right cosets of $G_{i}$ in $G$ and for $G_{i} x$ and $G_{j} y(x, y \in G, i, j \in I) G_{i} x \star G_{j} y$ whenever $G_{i} x \cap G_{j} y \neq \emptyset$. Also by letting $G$ act upon $\Gamma(G, \mathcal{F})$ by right multiplication we see that $\Gamma(G, \mathcal{F})$ is a flag transitive geometry for $G$. Moreover $\Gamma$ and $\Gamma(G, \mathcal{F})$ are isomorphic geometries for $G$. So we shall be studying geometries of the form $\Gamma(G, \mathcal{F})$, where $G \cong M_{23}$ and $G_{i}$ is a maximal subgroup of $G$ for all $i \in I$.

A numerical summary of our results is given in

Theorem 1. Suppose $G \cong M_{23}$ and $\Gamma$ is residually connected flag transitive geometry for $G$ with $G_{i}$ is a maximal subgroup of $G$. If $\Gamma$ has rank 3 , then up to conjugacy in $A u t G$, then there are 1050 possibilities for $\Gamma$.

In Section 2, we give explicit descriptions of these geometries making heavy use of the degree 23 permutation representation for $M_{23}$. We use GAP for some part of our calculation see [6]; especially calculation for $23: 11$.

Beukenhout, in [1], sought to give a wider view of geometries so as to encompass configurations observed in the finite sporadic simple groups. An outgrowth of this has been attemps to catalogue various subcollections of geometries for the finite sporadic simple groups (and other related groups). So - called minimal parabolic geometries and maximal 2-local geometries were investigated in [17] and [18] while geometries satisfying certain additional conditions for a number of (relatively) small order simple groups have been exhaustively examined. See, for example, [2], [5],[11], [12], [13], [14], [15], [16] and [19]. the results in most of these papers were obtained using various computer algebra systems. All rank2 and rank 3 residually connected geometries of $M_{22}$ (the Mathieu Group of degree 22) were investigated in [8]. Kilic, in [9], calculated all rank 2 geometries for the Mathieu group $M_{24}$. Again Kilic, in [10], calculated all rank 2 geometries for the Mathieu group $M_{23}$ for which object stabilizers are maximal subgroups. Now we determine all rank 3 residually connected flag transitive geometries for the Mathieu group $M_{23}$ (the Mathieu Group of degree 23) whose object stabilizer are maximal subgroups.

In this paper we shall use the result of all rank 2 geometries of $M_{23}$ calculated in $[10]$.

For the remainder of this paper $G$ will denote $M_{23}$, the Mathieu Group of degree 23. Also $\Omega$ will denote a 24 element set possessing the Steiner system $S(24,8,5)$ as described by Curtis's MOG [4]. We will follow the notation of [4]. 


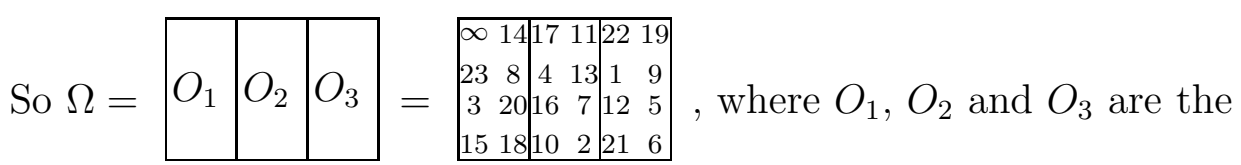

heavy bricks of the MOG. Here $M_{24}$ is the Mathieu group of degree 24 which leaves invariant the Steiner system $S(24,8,5)$ on $\Omega$. Set $\Lambda=\Omega \backslash\{\infty\}$

An octad of $\Omega$ is just an 8 -element block of the Steiner system and a subset of $\Omega$ is called a dodecad if it is the symmetric difference of two octads of $\Omega$ which intersect in a set of size two. Corresponding to each 4 points of $\Omega$ there is a partition of the 24 points into 6 tetrads with the property that the union of any two tetrads is an octad, this configuration will be called a sextet. The following sets will appear when we describe geometries for $G$.

(i) $\mathcal{D}=\{X \subseteq \Lambda|| X \mid=2\}$ (duads of $\Lambda$ ).

(ii) $\mathcal{H}=\{X \subseteq \Lambda \mid X \cup\{\infty\}$ is an octad of $\Omega\}$ (heptads of $\Lambda$ ).

(iii) $\mathcal{O}=\{X \subseteq \Lambda \mid X$ is an octad of $\Omega\}$ (octads of $\Lambda$ ).

(iv) $\mathcal{D}_{\mathrm{o}}=\{X \subseteq \Lambda \mid X$ is a dodecad of $\Omega\}$ (dodecads of $\Lambda$ ).

(v) $\mathcal{S}=\left\{X_{i} \subseteq \Omega|| X_{i} \mid=4\right.$ (for each $i \in I$ ), $X_{i} \cup X_{j}$ is an octad $(i \neq j$ ) and $\left.\Omega=\bigcup_{i \in I} X_{i}, i \in I=\{1 \ldots 6\}\right\}$ (sextets of $\Omega$ ).

From the [3], the conjugacy classes of the maximal subgroups of $G$ are as follows:

\begin{tabular}{|l|l|l|l|}
\hline Order & Index & $M_{i}$ & Description \\
\hline 443520 & 23 & $M_{1} \cong M_{22}$ & $M_{1}=\operatorname{Stab}_{G}\{a\}, a \in \Lambda$ \\
40320 & 253 & $M_{2} \cong L_{3}(4): 2 b$ & $M_{2}=\operatorname{Stab}_{G}\{X\}, X \in \mathcal{D}$ \\
40320 & 253 & $M_{3} \cong 2^{4}: A_{7}$ & $M_{3}=\operatorname{Stab}_{G}\{X\}, X \in \mathcal{H}$ \\
20160 & 506 & $M_{4} \cong A_{8}$ & $M_{4}=\operatorname{Stab}_{G}\{X\}, X \in \mathcal{O}$ \\
7920 & 1288 & $M_{5} \cong M_{11}$ & $M_{5}=\operatorname{Stab}_{G}\{X\}, X \in \mathcal{D}_{\mathrm{o}}$ \\
5760 & 1771 & $M_{6} \cong 2^{4}:\left(3 \times A_{5}\right): 2$ & $M_{6}=\operatorname{Stab}_{G}\{X\}, X \in \mathcal{S}$ \\
253 & 40320 & $M_{7} \cong 23: 11$ & \\
\hline
\end{tabular}

For $i \in\{1, \ldots, 7\}$, we let $\mathfrak{M}_{\mathrm{i}}$ denote the conjugacy class of $M_{i}, M_{i}$ as given in the previous table. We also set $\mathfrak{M}=\bigcup_{i=1}^{7} \mathfrak{M}_{i}$; so $\mathfrak{M}$ consist of all maximal subgroups of $G$. In [4] and [9], we can find further information about 23:11. Also put $\mathfrak{X}=\Lambda \cup \mathcal{D} \cup \mathcal{H} \cup \mathcal{O} \cup \mathcal{D}_{\mathrm{o}} \cup \mathcal{S}$.

Suppose $G_{1}$ and $G_{2}$ are maximal subgroups of $G$ with $G_{1} \neq G_{2}$. Set $G_{12}=$ $G_{1} \cap G_{2}$. We use $\mathfrak{M}_{\mathrm{ij}}(t)$ to describe $\left\{G_{1}, G_{2}, G_{1} \cap G_{2}\right\}$ according to the following scheme: $G_{1} \in \mathfrak{M}_{\mathrm{i}}, G_{2} \in \mathfrak{M}_{\mathrm{j}}$ (and so $G_{1}=\operatorname{Stab}_{G}\left(X_{1}\right)$ and $G_{2}=\operatorname{Stab}_{G}\left(X_{2}\right)$ for some appropriate subsets $X_{1}$ and $X_{2}$ of $\Lambda$ in $\left.\mathfrak{X}\right)$ with $\left|X_{1} \cap X_{2}\right|=t$. When listing up the rank 2 geometries of $G$ in [10] the notation $\mathfrak{M}_{\mathrm{ij}}(t)$ is not sufficient enough to describe the geometries up to conjugacy in Aut $G$. All calculations in $2^{4}:\left(3 \times A_{5}\right): 2$ and $23: 11$ we can not use this notation, we shall use the following notation; $\mathfrak{M}_{46}(1)$ means the first case of the intersection of octad and 
sextet, $\mathfrak{M}_{46}(2)$ means the second case of the intersection of octad and sextet. In [10], we shall find more information about it.

Now suppose we have three distinct maximal subgroups of $G ; G_{1}, G_{2}$ and $G_{3}$. We shall use use $G_{12}, G_{13}, G_{23}$ and $G_{123}$ to denote, respectively $G_{1} \cap G_{2}$, $G_{1} \cap G_{3}, G_{2} \cap G_{3}$ and $G_{1} \cap G_{2} \cap G_{3}$. We extend the above notation using $\mathfrak{M}_{i j k}\left(t_{i j}, t_{i k}, t_{j k}\right)$ to indicate that $G_{1} \in \mathfrak{M}_{i}, G_{2} \in \mathfrak{M}_{j}, G_{3} \in \mathfrak{M}_{k}$ with $\mid X_{i} \cap$ $X_{j}\left|=t_{i j},\right| X_{i} \cap X_{k} \mid=t_{i k}$ and $\left|X_{j} \cap X_{k}\right|=t_{j k}$. (Here $G_{1}=\operatorname{Stab}_{G}\left(X_{i}\right), G_{2}=$ $\operatorname{Stab}_{G}\left(X_{j}\right), G_{3}=\operatorname{Stab}_{G}\left(X_{k}\right)$ for suitable $X_{i}, X_{j}$ and $X_{k}$ of $\left.\Lambda \in \mathfrak{X}\right)$. Again we run into the possibility that in some instances, we need further subdivide these cases, and we do this using the ad hoc notation $\mathfrak{M}_{i j k}\left(t_{i j}, t_{i k}, t_{j k}: l\right)$ where $l \in\{1,2,3,4\}$. We note that if two or more $i, j$ and $k$ are equal, apparently different parameters $t_{i j}, t_{i k}, t_{j k}$ may describe the same situation. For examle $\mathfrak{M}_{344}(2,0,4)$ and $\mathfrak{M}_{344}(0,2,4)$ describe the same configuration as do $\mathfrak{M}_{333}(3,1,1)$ and $\mathfrak{M}_{333}(1,3,1)$.

We remark that the geometry $\Gamma(G, \mathcal{F})$ where $\mathcal{F}=\left\{G_{1}, G_{2}, G_{3}\right\}$ is residually connected if and only if $G_{1}=<G_{12}, G_{13}>, G_{2}=<G_{12}, G_{23}>$ and $G_{3}=<$ $G_{13}, G_{23}>$.

Below we give certain subsets of $\Lambda$ which will be encountered frequently in our list.

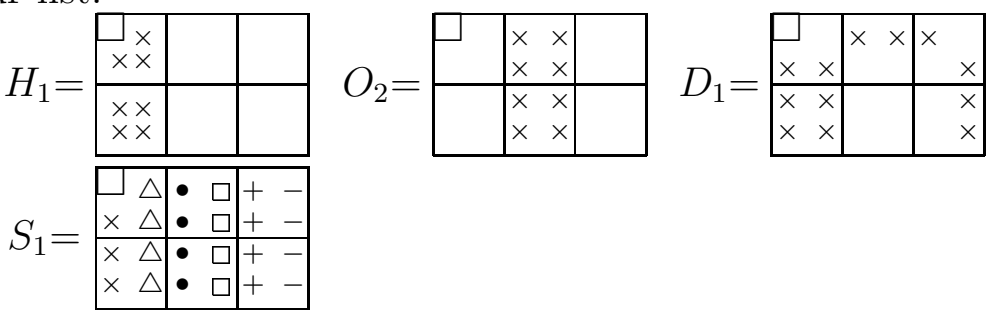

Our notation is as in [3] and [10] with the following addition: $F_{n}$ a Frobenious group of order $\mathrm{n}$ and $\left(S_{n} \times S_{m}\right)^{+}$is the group of even permutation in the permutation group $S_{n} \times S_{m}$.

\section{RANK 3 GEOMETRIES OF $M_{23}$}

Theorem 2. Up to conjugacy in Aut $G$ there are 1050 rank 3 residually connected geometries of $\Gamma=\Gamma\left(G,\left\{G_{1}, G_{2}, G_{3}\right\}\right)$ with $G_{1}, G_{2}, G_{3} \in \mathfrak{M}$. These together with the shape of $G_{123}$ are listed in the following table. 
On rank 3 residually connected geometries

\begin{tabular}{|c|c|c|c|}
\hline$\Gamma$ & $G_{123}$ & $\Gamma$ & $G_{123}$ \\
\hline $\mathfrak{M}_{111}(0,0,0)$ & $2^{4} . S L(2,4)$ & $\mathfrak{M}_{112}(0,0,1)$ & $2^{4} . S L(2,4)$ \\
\hline $\mathfrak{M}_{112}(0,0,0)$ & $2^{4} . S_{3}$ & $\mathfrak{M}_{113}(0,1,1)$ & $2^{4} \cdot A_{5}$ \\
\hline $\mathfrak{M}_{113}(0,1,0)$ & $A_{6}$ & $\mathfrak{M}_{113}(0,0,0)$ & $L_{3}(2)$ \\
\hline $\mathfrak{M}_{114}(0,1,1)$ & $A_{6}$ & $\mathfrak{M}_{114}(0,0,0)$ & $2^{4} \cdot S_{3}$ \\
\hline $\mathfrak{M}_{114}(0,0,1)$ & $L_{3}(2)$ & $\mathfrak{M}_{115}(0,1,1)$ & $A_{5}$ \\
\hline $\mathfrak{M}_{115}(0,1,0)$ & $A_{5}$ & $\mathfrak{M}_{115}(0,0,0)$ & $3^{2} \cdot Q_{8}$ \\
\hline $\mathfrak{M}_{116}(0,1,1)$ & $3^{2} .2$ & $\mathfrak{M}_{116}(0,2,2)$ & $2^{4} \cdot A_{5}$ \\
\hline$\overline{\mathfrak{M}_{117}(0,1,1)}$ & 1 & & \\
\hline $\mathfrak{M}_{122}(1,0,0)$ & $2^{4} . S_{3}$ & $\mathfrak{M}_{122}(0,0,0: 1)$ & $2^{4} .2^{2}$ \\
\hline $\mathfrak{M}_{122}(0,0,0: 2)$ & $D_{12}$ & $\mathfrak{M}_{122}(0,0,1)$ & $2^{4} .3$ \\
\hline$\overline{\bar{M}_{123}(1,1,2)}$ & $\overline{2^{4} . A_{5}}$ & $\overline{\mathfrak{M}_{123}(0,1,2)}$ & $\overline{2^{4} . S_{4}}$ \\
\hline $\mathfrak{M}_{123}(1,0,0)$ & $L_{3}(2)$ & $\mathfrak{M}_{123}(0,0,2)$ & $S_{5}$ \\
\hline $\mathfrak{M}_{123}(0,1,1)$ & $A_{5}$ & $\mathfrak{M}_{123}(0,1,0)$ & $2^{3} \cdot S_{3}$ \\
\hline $\mathfrak{M}_{123}(0,0,0)$ & $S_{4}$ & $\mathfrak{M}_{123}(0,0,1)$ & $S_{4}$ \\
\hline$\overline{\mathfrak{M}_{124}(1,1,2)}$ & $\overline{A_{6}}$ & $\overline{\mathfrak{M}_{124}(0,1,2)}$ & $\overline{S_{5}}$ \\
\hline $\mathfrak{M}_{124}(1,0,0)$ & $2^{4} . S_{3}$ & $\mathfrak{M}_{124}(0,0,2)$ & $2^{2} \cdot D_{12}$ \\
\hline $\mathfrak{M}_{124}(0,1,0)$ & $S_{4}$ & $\mathfrak{M}_{124}(0,1,1)$ & $S_{4}$ \\
\hline $\mathfrak{M}_{124}(0,0,0)$ & $2^{3} .2$ & $\mathfrak{M}_{124}(0,0,1)$ & $A_{4}$ \\
\hline$\overline{\mathfrak{M}_{125}(1,1,0)}$ & $\overline{\bar{M} M_{9}}$ & $\overline{\mathfrak{M}_{125}(1,0,2)}$ & $\overline{A_{5}}$ \\
\hline $\mathfrak{M}_{125}(0,1,2: 1)$ & $S_{4}$ & $\mathfrak{M}_{125}(0,1,2: 2)$ & $F_{20}$ \\
\hline $\mathfrak{M}_{125}(0,1,0)$ & $Q_{8.2}$ & $\mathfrak{M}_{125}(0,0,0)$ & $D_{12}$ \\
\hline $\mathfrak{M}_{125}(0,0,2)$ & $D_{12}$ & $\mathfrak{M}_{125}(0,0,1: 1)$ & $A_{4}$ \\
\hline $\mathfrak{M}_{125}(0,0,1: 2)$ & $\overline{D_{10}}$ & $\mathfrak{M}_{125}(0,1,1)$ & $S_{3}$ \\
\hline$\overline{\mathfrak{M}}_{126}(1,2,3)$ & $\overline{2^{4} . A_{5}}$ & $\overline{\mathfrak{M}_{126}(1,1,3)}$ & $\overline{2^{4} . S_{3}}$ \\
\hline $\mathfrak{M}_{126}(0,1,3)$ & $2^{4} . S_{3}$ & $\mathfrak{M}_{126}(0,2,1)$ & $2^{3} \cdot D_{8}$ \\
\hline $\mathfrak{M}_{126}(0,2,4)$ & $2^{4} .3$ & $\mathfrak{M}_{126}(1,1,2)$ & $3^{2} .2$ \\
\hline $\mathfrak{M}_{126}(0,1,1)$ & $D_{12}$ & $\mathfrak{M}_{126}(0,2,2)$ & $D_{12}$ \\
\hline $\mathfrak{M}_{126}(0,1,2: 1)$ & $D_{12}$ & $\mathfrak{M}_{126}(0,1,2: 2)$ & $S_{3}$ \\
\hline $\mathfrak{M}_{126}(0,1,2: 3)$ & $2^{2}$ & $\mathfrak{M}_{126}(0,1,4)$ & $S_{3}$ \\
\hline $\mathfrak{M}_{133}(1,1,3)$ & $2^{4} . S_{3}$ & $\mathfrak{M}_{133}(0,1,3)$ & $\left(S_{3} \times S_{4}\right)^{+}$ \\
\hline $\mathfrak{M}_{133}(1,0,1)$ & $A_{5}$ & $\mathfrak{M}_{133}(0,0,1)$ & $3^{2} .4$ \\
\hline $\mathfrak{M}_{133}(0,0,3)$ & $S_{4}$ & & \\
\hline$\overline{\mathfrak{M}_{134}(1,0,0)}$ & $\overline{2^{3} . S_{4}}$ & $\mathfrak{M}_{134}(0,0,0)$ & $L_{3}(2)$ \\
\hline \multirow[t]{2}{*}{$\mathfrak{M}_{134}(0,1,0)$} & $L_{3}(2)$ & $\mathfrak{M}_{134}(1,0,4)$ & $2^{2} . S_{4}$ \\
\hline & & $\mathfrak{M}_{134}(1,1,4)$ & $\left(S_{3} \times S_{4}\right)^{+}$ \\
\hline $\mathfrak{M}_{134}(0,1,4)$ & $\left(S_{3} \times S_{4}\right)^{+}$ & $\mathfrak{M}_{134}(1,1,2)$ & $A_{5}$ \\
\hline $\mathfrak{M}_{134}(0,0,4)$ & $S_{4}$ & $\mathfrak{M}_{134}(1,0,2)$ & $S_{4}$ \\
\hline $\mathfrak{M}_{134}(0,1,2)$ & $F_{20}$ & $\mathfrak{M}_{134}(0,0,2)$ & $D_{12}$ \\
\hline$\overline{\mathfrak{M}_{135}(1,0,6)}$ & $\overline{A_{5}}$ & $\overline{\mathfrak{M}_{135}(0,0,6)}$ & $\overline{A_{5}}$ \\
\hline $\mathfrak{M}_{135}(1,0,2)$ & $A_{5}$ & $\mathfrak{M}_{135}(0,1,6)$ & $3^{2} .4$ \\
\hline $\mathfrak{M}_{135}(1,1,2)$ & $S_{4}$ & $\mathfrak{M}_{135}(0,1,2)$ & $F_{20}$ \\
\hline $\mathfrak{M}_{135}(1,1,4)$ & $M_{8} .2$ & $\mathfrak{M}_{135}(1,0,4)$ & $D_{12}$ \\
\hline
\end{tabular}




\begin{tabular}{|c|c|c|c|}
\hline $\mathfrak{M}_{135}(0,0,2)$ & $D_{12}$ & $\mathfrak{M}_{135}(0,0,4)$ & $S_{3}$ \\
\hline $\mathfrak{M}_{135}(0,1,4)$ & $S_{3}$ & & \\
\hline$\overline{\mathfrak{M}_{136}(1,2,1)}$ & $\overline{2^{4} . S_{4}}$ & $\overline{\mathfrak{M}_{136}(0,1,1)}$ & $\overline{\left(S_{3} \times S_{4}\right)^{+}}$ \\
\hline $\mathfrak{M}_{136}(1,2,4)$ & $A_{5}$ & $\mathfrak{M}_{136}(0,2,2)$ & $S_{4}$ \\
\hline $\mathfrak{M}_{136}(0,2,3)$ & $S_{4}$ & $\mathfrak{M}_{136}(1,1,4)$ & $S_{4}$ \\
\hline $\mathfrak{M}_{136}(1,1,3)$ & $3^{2} .2$ & $\mathfrak{M}_{136}(1,1,2)$ & $2^{3}$ \\
\hline $\mathfrak{M}_{136}(0,1,2: 1)$ & $D_{8}$ & $\mathfrak{M}_{136}(0,1,2: 2)$ & $S_{3}$ \\
\hline $\mathfrak{M}_{136}(0,1,4)$ & $\overline{D_{8}}$ & $\mathfrak{M}_{136}(0,1,3)$ & $S_{3}$ \\
\hline$\overline{\mathfrak{M}_{144}(0,0,0)}$ & $\overline{2^{3} \cdot S_{4}}$ & $\overline{\mathfrak{M}_{144}(1,0,0)}$ & 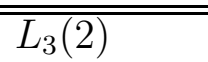 \\
\hline $\mathfrak{M}_{144}(0,0,4: 1)$ & $2^{2} . D_{8}$ & $\mathfrak{M}_{144}(0,0,4: 2)$ & $A_{4}$ \\
\hline $\mathfrak{M}_{144}(1,1,2)$ & $3^{2} .4$ & $\mathfrak{M}_{144}(0,1,4)$ & $S_{4}$ \\
\hline $\mathfrak{M}_{144}(1,1,4)$ & $S_{4}$ & $\mathfrak{M}_{144}(0,1,2)$ & $D_{12}$ \\
\hline $\mathfrak{M}_{144}(0,0,2)$ & $D_{8}$ & & \\
\hline$\overline{\mathfrak{M}_{145}(1,0,2)}$ & $\overline{\overline{A_{5}}}$ & $\mathfrak{M}_{145}(1,1,6)$ & $3^{2} .4$ \\
\hline $\mathfrak{M}_{145}(0,1,2)$ & $S_{4}$ & $\mathfrak{M}_{145}(1,1,2)$ & $F_{20}$ \\
\hline $\mathfrak{M}_{145}(0,0,2)$ & $D_{12}$ & $\mathfrak{M}_{145}(0,0,6)$ & $D_{12}$ \\
\hline $\mathfrak{M}_{145}(1,0,6)$ & $D_{12}$ & $\mathfrak{M}_{145}(0,0,4: 1)$ & $A_{4}$ \\
\hline $\mathfrak{M}_{145}(0,0,4: 2)$ & $2^{2}$ & $\mathfrak{M}_{145}(0,1,6)$ & $D_{8}$ \\
\hline $\mathfrak{M}_{145}(1,0,4)$ & $S_{3}$ & $\mathfrak{M}_{145}(1,1,4)$ & $S_{3}$ \\
\hline $\mathfrak{M}_{145}(0,1,4)$ & 4 & & \\
\hline$\overline{\mathfrak{M}_{146}(0,2,1)}$ & $\overline{2^{4} \cdot D_{12}}$ & $\overline{\mathfrak{M}_{146}(1,2,4)}$ & $S_{5}$ \\
\hline $\mathfrak{M}_{146}(0,1,1)$ & $2^{4} \cdot S_{3}$ & $\mathfrak{M}_{146}(1,1,1)$ & $\left(S_{3} \times S_{4}\right)^{+}$ \\
\hline $\mathfrak{M}_{146}(1,1,4)$ & $\left(S_{3} \times S_{4}\right)^{+}$ & $\mathfrak{M}_{146}(0,1,4)$ & $S_{4}$ \\
\hline $\mathfrak{M}_{146}(1,2,5)$ & $S_{4}$ & $\mathfrak{M}_{146}(0,2,2)$ & $2^{3} .2$ \\
\hline $\mathfrak{M}_{146}(0,2,3)$ & $A_{4}$ & $\mathfrak{M}_{146}(1,1,5)$ & $D_{8}$ \\
\hline $\mathfrak{M}_{146}(0,1,5: 1)$ & $2^{3}$ & $\mathfrak{M}_{146}(1,1,2)$ & $S_{3}$ \\
\hline $\mathfrak{M}_{146}(0,1,5: 2)$ & $S_{3}$ & $\mathfrak{M}_{146}(0,1,2)$ & $S_{3}$ \\
\hline $\mathfrak{M}_{146}(1,1,3)$ & $S_{3}$ & $\mathfrak{M}_{146}(0,1,3)$ & 2 \\
\hline$\overline{\mathfrak{M}_{155}(1,1,4)}$ & 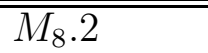 & $\overline{\mathfrak{M}_{155}(0,0,4)}$ & $\overline{D_{12}}$ \\
\hline $\mathfrak{M}_{155}(0,0,8: 1)$ & $A_{4}$ & $\mathfrak{M}_{155}(0,0,8: 2)$ & $2^{2}$ \\
\hline $\mathfrak{M}_{155}(1,0,4)$ & $S_{3}$ & $\mathfrak{M}_{155}(0,1,8)$ & $S_{3}$ \\
\hline $\mathfrak{M}_{155}(1,1,8)$ & 4 & $\mathfrak{M}_{155}(1,0,6)$ & 2 \\
\hline $\mathfrak{M}_{155}(1,1,6)$ & 2 & $\mathfrak{M}_{155}(0,0,6)$ & 2 \\
\hline $\bar{M}_{156}(1,1,1: 1)$ & $\overline{3^{2} .2}$ & $\overline{\bar{M}_{156}(1,1,1: 2)}$ & $\overline{2^{2}}$ \\
\hline $\mathfrak{M}_{156}(1,2,5)$ & $M_{8} .2$ & $\mathfrak{M}_{156}(0,2,1)$ & $D_{12}$ \\
\hline $\mathfrak{M}_{156}(0,1,1: 1)$ & $D_{12}$ & $\mathfrak{M}_{156}(0,1,1: 2)$ & $S_{3}$ \\
\hline $\mathfrak{M}_{156}(0,2,4)$ & $\overline{A_{4}}$ & $\mathfrak{M}_{156}(0,2,3)$ & $D_{10}$ \\
\hline $\mathfrak{M}_{156}(1,2,2)$ & $S_{3}$ & $\mathfrak{M}_{156}(0,1,2: 1)$ & $S_{3}$ \\
\hline $\mathfrak{M}_{156}(0,1,2: 2)$ & $2^{2}$ & $\mathfrak{M}_{156}(0,1,2: 3)$ & 2 \\
\hline $\mathfrak{M}_{156}(0,1,5)$ & $S_{3}$ & $\mathfrak{M}_{156}(1,1,5)$ & $S_{3}$ \\
\hline $\mathfrak{M}_{156}(1,1,2: 1)$ & $2^{2}$ & $\mathfrak{M}_{156}(1,1,2: 2)$ & 2 \\
\hline $\mathfrak{M}_{156}(0,1,4)$ & $\frac{2^{2}}{3}$ & $\mathfrak{M}_{156}(1,1,3)$ & 4 \\
\hline
\end{tabular}




\begin{tabular}{|c|c|c|c|}
\hline $\mathfrak{M}_{156}(1,1,4)$ & 4 & $\mathfrak{M}_{156}(0,1,3)$ & 2 \\
\hline $\mathfrak{M}_{157}(0,1,1)$ & 1 & $\mathfrak{M}_{157}(1,1,1)$ & 1 \\
\hline$\overline{\mathfrak{M}}_{166}(2,2,4)$ & $22^{4} .3$ & $\bar{M}_{166}(1,1,2: 1)$ & $3^{2} .2$ \\
\hline $\mathfrak{M}_{166}(1,1,2: 2)$ & $S_{3}$ & $\mathfrak{M}_{166}(1,1,2: 3)$ & $2^{2}$ \\
\hline $\mathfrak{M}_{166}(1,1,5)$ & $3^{2} .2$ & $\mathfrak{M}_{166}(1,2,2)$ & $D_{12}$ \\
\hline $\mathfrak{M}_{166}(1,1,4)$ & $S_{3}$ & $\mathfrak{M}_{166}(2,1,1: 1)$ & $S_{3}$ \\
\hline $\mathfrak{M}_{166}(2,1,1: 2)$ & $2^{2}$ & $\mathfrak{M}_{166}(1,1,3)$ & $2^{2}$ \\
\hline $\mathfrak{M}_{166}(1,1,1)$ & 2 & & \\
\hline$\overline{\mathfrak{M}_{177}(1,1,2)}$ & 1 & & \\
\hline$* * * * * * * * * * * *$ & $* * * * *$ & $* * * * * * * * * * * *$ & $* * * * *$ \\
\hline $\mathfrak{M}_{222}(0,0,0: 1)$ & $2^{3}$ & $\mathfrak{M}_{222}(0,0,0: 2)$ & $2^{2}$ \\
\hline $\mathfrak{M}_{222}(0,1,0)$ & $S_{3}$ & & \\
\hline$\overline{\mathfrak{M}_{223}(1,2,2)}$ & $\overline{2^{4} . A_{4}}$ & $\mathfrak{M}_{223}(0,2,1)$ & $S_{4}$ \\
\hline $\mathfrak{M}_{223}(0,2,0)$ & $2^{3} .2$ & $\mathfrak{M}_{223}(1,0,0)$ & $A_{4}$ \\
\hline $\mathfrak{M}_{223}(0,1,0: 1)$ & $D_{8}$ & $\mathfrak{M}_{223}(0,1,0: 2)$ & $S_{3}$ \\
\hline $\mathfrak{M}_{223}(0,0,0)$ & $2^{2}$ & $\mathfrak{M}_{223}(0,1,1)$ & $2^{2}$ \\
\hline $\mathfrak{M}_{224}(1,2,2)$ & $A_{5}$ & $\mathfrak{M}_{224}(0,2,2)$ & $2 \times S_{4}$ \\
\hline $\mathfrak{M}_{224}(0,0,2: 1)$ & $D_{8} \times 2$ & $\mathfrak{M}_{224}(0,2,0: 2)$ & $D_{12}$ \\
\hline $\mathfrak{M}_{224}(0,0,0: 1)$ & $2 \times D_{8}$ & $\mathfrak{M}_{224}(0,0,0: 2)$ & $2^{2}$ \\
\hline $\mathfrak{M}_{224}(0,1,2)$ & $D_{8}$ & $\mathfrak{M}_{224}(1,0,0)$ & $2^{3}$ \\
\hline $\mathfrak{M}_{224}(0,1,1: 1)$ & $2^{2}$ & $\mathfrak{M}_{224}(0,1,1: 2)$ & 3 \\
\hline $\mathfrak{M}_{224}(0,0,1)$ & 2 & & \\
\hline $\mathfrak{M}_{225}(0,2,2: 1)$ & $D_{8}$ & $\mathfrak{M}_{225}(0,2,2: 2)$ & $2^{2}$ \\
\hline $\mathfrak{M}_{225}(0,2,0: 1)$ & $D_{8}$ & $\mathfrak{M}_{225}(0,2,0: 2)$ & 4 \\
\hline $\mathfrak{M}_{225}(1,0,0)$ & $\overline{Q_{8}}$ & $\mathfrak{M}_{225}(1,2,2)$ & $S_{3}$ \\
\hline $\mathfrak{M}_{225}(0,0,0)$ & $2^{2}$ & $\mathfrak{M}_{225}(0,1,1)$ & 3 \\
\hline $\mathfrak{M}_{225}(0,0,1)$ & 2 & $\mathfrak{M}_{225}(0,2,1)$ & 2 \\
\hline $\mathfrak{M}_{225}(0,1,1)$ & 2 & & \\
\hline$\overline{\mathfrak{M}}_{226}(0,3,2)$ & $\overline{D_{12}}$ & $\mathfrak{M}_{226}(0,1,1)$ & $2^{3}$ \\
\hline $\mathfrak{M}_{226}(0,1,2: 1)$ & $S_{3}$ & $\mathfrak{M}_{226}(0,1,2: 2)$ & $2^{2}$ \\
\hline $\mathfrak{M}_{226}(1,1,2)$ & $S_{3}$ & $\mathfrak{M}_{226}(0,2,4: 1)$ & $2^{2}$ \\
\hline $\mathfrak{M}_{226}(0,2,4: 2)$ & 2 & $\mathfrak{M}_{226}(0,1,4)$ & $2^{2}$ \\
\hline $\mathfrak{M}_{226}(0,4,4)$ & 3 & $\mathfrak{M}_{226}(0,2,2: 1)$ & 2 \\
\hline $\mathfrak{M}_{226}(0,2,2: 2)$ & 1 & $\mathfrak{M}_{226}(1,2,2)$ & 2 \\
\hline $\mathfrak{M}_{233}(2,2,3)$ & $2^{4} . S_{3}$ & $\mathfrak{M}_{233}(2,0,3)$ & 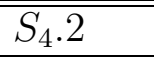 \\
\hline $\mathfrak{M}_{233}(2,0,1)$ & $S_{4}$ & $\mathfrak{M}_{233}(2,1,3)$ & $S_{4}$ \\
\hline $\mathfrak{M}_{233}(0,0,3: 1)$ & $2^{3} .2$ & $\mathfrak{M}_{233}(0,0,3: 2)$ & $\overline{S_{3}}$ \\
\hline $\mathfrak{M}_{233}(0,0,1)$ & $D_{8}$ & $\mathfrak{M}_{233}(1,0,3)$ & $S_{3}$ \\
\hline $\mathfrak{M}_{233}(1,0,1)$ & $S_{3}$ & & \\
\hline$\overline{\mathfrak{M}_{234}(2,0,0)}$ & $\overline{2^{3} \cdot D_{8}}$ & $\mathfrak{M}_{234}(2,2,4)$ & $S_{4} \times 2$ \\
\hline $\mathfrak{M}_{234}(0,2,4)$ & $S_{4} \times 2$ & $\mathfrak{M}_{234}(0,0,0)$ & $S_{4} .2$ \\
\hline
\end{tabular}




\begin{tabular}{|c|c|c|c|}
\hline $\mathfrak{M}_{234}(0,2,0)$ & $S_{4.2}$ & $\mathfrak{M}_{234}(2,1,4)$ & $S_{4}$ \\
\hline $\mathfrak{M}_{234}(1,0,0)$ & $S_{4}$ & $\mathfrak{M}_{234}(0,1,0)$ & $F_{21}$ \\
\hline $\mathfrak{M}_{234}(1,2,4)$ & $3^{2} .2$ & $\mathfrak{M}_{234}(0,0,4: 1)$ & $2 \times D_{8}$ \\
\hline $\mathfrak{M}_{234}(0,0,4: 2)$ & $S_{3}$ & $\mathfrak{M}_{234}(2,1,2)$ & $A_{4}$ \\
\hline $\mathfrak{M}_{234}(2,0,2)$ & $D_{12}$ & $\mathfrak{M}_{234}(1,2,2)$ & $D_{10}$ \\
\hline $\mathfrak{M}_{234}(0,2,2)$ & $D_{8}$ & $\mathfrak{M}_{234}(1,0,4: 1)$ & $D_{8}$ \\
\hline $\mathfrak{M}_{234}(1,0,4: 2)$ & $S_{3}$ & $\mathfrak{M}_{234}(0,0,2: 1)$ & $\overline{D_{8}}$ \\
\hline $\mathfrak{M}_{234}(0,0,2: 2)$ & $\frac{2^{2}}{2}$ & $\mathfrak{M}_{234}(0,1,4)$ & $S_{3}$ \\
\hline $\mathfrak{M}_{234}(1,0,2: 1)$ & $S_{3}$ & $\mathfrak{M}_{234}(1,0,2: 2)$ & $2^{2}$ \\
\hline $\mathfrak{M}_{234}(0,1,2)$ & 2 & & \\
\hline$\overline{\mathfrak{M}_{235}(2,2,6)}$ & $\overline{S_{4}}$ & $\mathfrak{M}_{235}(0,2,6)$ & $S_{4}$ \\
\hline $\mathfrak{M}_{235}(2,0,4)$ & $\overline{D_{16}}$ & $\mathfrak{M}_{235}(2,1,2)$ & $A_{4}$ \\
\hline $\mathfrak{M}_{235}(1,2,6)$ & $D_{10}$ & $\mathfrak{M}_{235}(0,0,6)$ & $D_{8}$ \\
\hline $\mathfrak{M}_{235}(0,0,2)$ & $D_{8}$ & $\mathfrak{M}_{235}(2,2,4)$ & $D_{8}$ \\
\hline $\mathfrak{M}_{235}(0,2,2: 1)$ & $D_{8}$ & $\mathfrak{M}_{235}(0,2,2: 2)$ & $2^{2}$ \\
\hline $\mathfrak{M}_{235}(1,2,2)$ & $S_{3}$ & $\mathfrak{M}_{235}(1,2,4: 1)$ & $S_{3}$ \\
\hline $\mathfrak{M}_{235}(1,2,4: 2)$ & 2 & $\mathfrak{M}_{235}(0,1,6)$ & $S_{3}$ \\
\hline $\mathfrak{M}_{235}(1,0,2)$ & 4 & $\mathfrak{M}_{235}(2,1,4)$ & $2^{2}$ \\
\hline $\mathfrak{M}_{235}(0,1,4: 1)$ & 3 & $\mathfrak{M}_{235}(0,1,4: 2)$ & 2 \\
\hline $\mathfrak{M}_{235}(0,1,2)$ & 2 & $\mathfrak{M}_{235}(0,2,4)$ & 2 \\
\hline $\mathfrak{M}_{235}(0,0,4)$ & 2 & $\mathfrak{M}_{235}(1,0,4)$ & 2 \\
\hline$\overline{\mathfrak{M}_{236}(2,3,1)}$ & $2^{4} . S_{4}$ & $\overline{\mathfrak{M}_{236}(0,1,1)}$ & " \\
\hline $\mathfrak{M}_{236}(0,3,3)$ & $S_{4}$ & $\mathfrak{M}_{236}(1,2,1)$ & $3^{2} .2$ \\
\hline $\mathfrak{M}_{236}(2,1,2)$ & $2 \times D_{8}$ & $\mathfrak{M}_{236}(2,4,4)$ & $A_{4}$ \\
\hline $\mathfrak{M}_{236}(2,2,4)$ & $D_{12}$ & $\mathfrak{M}_{236}(0,2,1)$ & $D_{12}$ \\
\hline $\mathfrak{M}_{236}(0,1,4)$ & $D_{8}$ & $\mathfrak{M}_{236}(1,1,4)$ & $D_{8}$ \\
\hline $\mathfrak{M}_{236}(2,2,3)$ & $S_{3}$ & $\mathfrak{M}_{236}(0,1,3)$ & $S_{3}$ \\
\hline $\mathfrak{M}_{236}(1,1,3)$ & $S_{3}$ & $\mathfrak{M}_{236}(0,2,3: 1)$ & $S_{3}$ \\
\hline $\mathfrak{M}_{236}(0,2,3: 2)$ & $2^{2}$ & $\mathfrak{M}_{236}(0,2,3: 3)$ & 2 \\
\hline $\mathfrak{M}_{236}(0,4,2: 1)$ & $2^{2}$ & $\mathfrak{M}_{236}(0,4,2: 2)$ & 3 \\
\hline $\mathfrak{M}_{236}(2,2,2)$ & $2^{2}$ & $\mathfrak{M}_{236}(0,2,4: 1)$ & $2^{2}$ \\
\hline $\mathfrak{M}_{236}(0,2,4: 2)$ & 2 & $\mathfrak{M}_{236}(0,1,2)$ & $2^{2}$ \\
\hline $\mathfrak{M}_{236}(1,1,2)$ & $2^{2}$ & $\mathfrak{M}_{236}(1,2,3)$ & 2 \\
\hline $\mathfrak{M}_{236}(1,2,2)$ & 2 & $\mathfrak{M}_{236}(0,4,3)$ & 2 \\
\hline $\mathfrak{M}_{236}(1,2,4)$ & 2 & $\mathfrak{M}_{236}(0,2,2)$ & 1 \\
\hline $\mathfrak{M}_{244}(2,0,0)$ & $S_{4} \times 2$ & $\mathfrak{M}_{244}(1,0,0)$ & $S_{4}$ \\
\hline $\mathfrak{M}_{244}(2,2,4)$ & $2^{3} .2$ & $\mathfrak{M}_{244}(2,0,4)$ & $2^{3} .2$ \\
\hline $\mathfrak{M}_{244}(2,0,2: 1)$ & $D_{12}$ & $\mathfrak{M}_{244}(2,0,2: 2)$ & $D_{8}$ \\
\hline $\mathfrak{M}_{244}(0,0,4: 1)$ & $2^{3}$ & $\mathfrak{M}_{244}(0,0,4: 2)$ & $S_{3}$ \\
\hline $\mathfrak{M}_{244}(0,0,4: 3)$ & $2^{2}$ & $\mathfrak{M}_{244}(1,0,4: 1)$ & $D_{8}$ \\
\hline $\mathfrak{M}_{244}(0,1,4: 2)$ & 3 & $\mathfrak{M}_{244}(2,1,2)$ & $S_{3}$ \\
\hline $\mathfrak{M}_{244}(1,2,4)$ & $S_{3}$ & $\mathfrak{M}_{244}(1,0,2: 1)$ & $2^{2}$ \\
\hline
\end{tabular}




\begin{tabular}{|c|c|c|c|}
\hline $\mathfrak{M}_{244}(1,0,2: 2)$ & 2 & $\mathfrak{M}_{244}(0,0,2)$ & $2^{2}$ \\
\hline $\mathfrak{M}_{244}(1,1,2)$ & 2 & & \\
\hline$\overline{\mathfrak{M}}_{245}(2,2,6: 1)$ & $\overline{D_{12}}$ & $\mathfrak{M}_{245}(2,2,6: 2)$ & $D_{8}$ \\
\hline $\mathfrak{M}_{245}(0,2,6: 1)$ & $D_{12}$ & $\mathfrak{M}_{245}(0,2,6: 2)$ & $D_{8}$ \\
\hline $\mathfrak{M}_{245}(0,0,2)$ & $D_{12}$ & $\mathfrak{M}_{245}(2,1,2)$ & $D_{10}$ \\
\hline $\mathfrak{M}_{245}(0,2,2: 1)$ & $D_{8}$ & $\mathfrak{M}_{245}(0,2,2: 2)$ & $2^{2}$ \\
\hline $\mathfrak{M}_{245}(2,0,2)$ & $D_{8}$ & $\mathfrak{M}_{245}(2,1,4: 1)$ & $S_{3}$ \\
\hline $\mathfrak{M}_{245}(2,1,4: 2)$ & 2 & $\mathfrak{M}_{245}(1,2,2)$ & $S_{3}$ \\
\hline $\mathfrak{M}_{245}(2,1,6)$ & $S_{3}$ & $\mathfrak{M}_{245}(0,1,2: 1)$ & $S_{3}$ \\
\hline $\mathfrak{M}_{245}(0,1,2: 2)$ & $2^{2}$ & $\mathfrak{M}_{245}(0,0,4: 1)$ & 4 \\
\hline $\mathfrak{M}_{245}(0,0,4: 2)$ & 2 & $\mathfrak{M}_{245}(1,0,2)$ & 4 \\
\hline $\mathfrak{M}_{245}(1,0,6)$ & 4 & $\mathfrak{M}_{245}(1,0,4: 1)$ & 4 \\
\hline $\mathfrak{M}_{245}(1,0,4: 2)$ & 1 & $\mathfrak{M}_{245}(2,0,4)$ & $2^{2}$ \\
\hline $\mathfrak{M}_{245}(0,0,6)$ & $2^{2}$ & $\mathfrak{M}_{245}(0,1,6: 1)$ & $2^{2}$ \\
\hline $\mathfrak{M}_{245}(0,1,6: 2)$ & 2 & $\mathfrak{M}_{245}(2,2,4)$ & $2^{2}$ \\
\hline $\mathfrak{M}_{245}(1,2,4: 1)$ & 3 & $\mathfrak{M}_{245}(1,2,4: 2)$ & 2 \\
\hline $\mathfrak{M}_{245}(0,1,4: 1)$ & 2 & $\mathfrak{M}_{245}(0,1,4: 2)$ & 1 \\
\hline $\mathfrak{M}_{245}(1,2,6)$ & 2 & $\mathfrak{M}_{245}(0,2,4)$ & 2 \\
\hline$\overline{\mathfrak{M}_{246}(2,3,4)}$ & $S_{5}$ & $\overline{\mathfrak{M}_{246}(2,1,1)}$ & $S_{4.2}$ \\
\hline $\mathfrak{M}_{246}(0,1,1)$ & $2^{3} \cdot 2^{2}$ & $\mathfrak{M}_{246}(2,4,4)$ & $S_{4}$ \\
\hline $\mathfrak{M}_{246}(0,1,4)$ & $S_{4}$ & $\mathfrak{M}_{246}(1,1,4)$ & $S_{4}$ \\
\hline $\mathfrak{M}_{246}(0,4,1)$ & $2^{3} .2$ & $\mathfrak{M}_{246}(0,3,2)$ & $2^{3} .2$ \\
\hline $\mathfrak{M}_{246}(2,1,5)$ & $D_{8} \times 2$ & $\mathfrak{M}_{246}(0,2,1)$ & $D_{12}$ \\
\hline $\mathfrak{M}_{246}(2,1,2)$ & $D_{12}$ & $\mathfrak{M}_{246}(1,2,4)$ & $S_{3}$ \\
\hline $\mathfrak{M}_{246}(1,2,1)$ & $S_{3}$ & $\mathfrak{M}_{246}(0,2,2: 1)$ & $S_{3}$ \\
\hline $\mathfrak{M}_{246}(0,2,2: 2)$ & $2^{2}$ & $\mathfrak{M}_{246}(0,2,2: 3)$ & 2 \\
\hline $\mathfrak{M}_{246}(0,2,4)$ & $2^{2}$ & $\mathfrak{M}_{246}(2,2,3: 1)$ & $2^{2}$ \\
\hline $\mathfrak{M}_{246}(2,2,3: 2)$ & 2 & $\mathfrak{M}_{246}(0,1,5)$ & $2^{2}$ \\
\hline $\mathfrak{M}_{246}(1,1,5)$ & $2^{2}$ & $\mathfrak{M}_{246}(2,2,2)$ & $2^{2}$ \\
\hline $\mathfrak{M}_{246}(2,4,5)$ & $2^{2}$ & $\mathfrak{M}_{246}(2,2,5)$ & $2^{2}$ \\
\hline $\mathfrak{M}_{246}(1,1,2)$ & 3 & $\mathfrak{M}_{246}(0,4,2)$ & 2 \\
\hline $\mathfrak{M}_{246}(1,2,3: 1)$ & 2 & $\mathfrak{M}_{246}(1,2,3: 2)$ & 1 \\
\hline $\mathfrak{M}_{246}(0,1,3)$ & 2 & $\mathfrak{M}_{246}(1,2,5: 1)$ & 2 \\
\hline $\mathfrak{M}_{246}(1,2,5: 2)$ & 1 & $\mathfrak{M}_{246}(1,2,2: 1)$ & 2 \\
\hline $\mathfrak{M}_{246}(1,2,2: 2)$ & 1 & $\mathfrak{M}_{246}(0,2,3: 1)$ & 2 \\
\hline $\mathfrak{M}_{246}(0,2,3: 2)$ & 1 & $\mathfrak{M}_{246}(1,1,3)$ & 2 \\
\hline $\mathfrak{M}_{246}(0,2,5)$ & 2 & $\mathfrak{M}_{246}(0,4,3)$ & 1 \\
\hline $\mathfrak{M}_{255}(0,0,4)$ & $D_{16}$ & $\mathfrak{M}_{255}(0,0,8: 1)$ & $D_{8}$ \\
\hline $\mathfrak{M}_{255}(0,0,8: 2)$ & 4 & $\mathfrak{M}_{255}(0,0,8: 3)$ & 2 \\
\hline $\mathfrak{M}_{255}(2,2,4)$ & $D_{8}$ & $\mathfrak{M}_{255}(2,0,8)$ & 4 \\
\hline $\mathfrak{M}_{255}(2,1,8: 1)$ & 3 & $\mathfrak{M}_{255}(2,1,8: 2)$ & 2 \\
\hline $\mathfrak{M}_{255}(2,2,8)$ & 2 & $\mathfrak{M}_{255}(0,2,6)$ & 2 \\
\hline
\end{tabular}




\begin{tabular}{|c|c|c|c|}
\hline $\mathfrak{M}_{255}(0,1,6: 1)$ & 2 & $\mathfrak{M}_{255}(0,1,6: 2)$ & 1 \\
\hline $\mathfrak{M}_{255}(0,0,6)$ & 2 & $\mathfrak{M}_{255}(2,0,4)$ & 2 \\
\hline $\mathfrak{M}_{255}(1,2,6: 1)$ & 2 & $\mathfrak{M}_{255}(1,2,6: 2)$ & 1 \\
\hline $\mathfrak{M}_{255}(2,2,6)$ & 2 & $\mathfrak{M}_{255}(1,0,4)$ & 2 \\
\hline $\mathfrak{M}_{255}(2,1,4)$ & 2 & $\mathfrak{M}_{255}(1,0,8)$ & 1 \\
\hline$\overline{\mathfrak{M}_{256}(0,3,5)}$ & $Q_{8.2}$ & 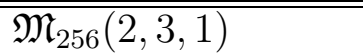 & $D_{12}$ \\
\hline $\mathfrak{M}_{256}(0,1,5)$ & $D_{12}$ & $\mathfrak{M}_{256}(0,1,4)$ & $D_{8}$ \\
\hline $\mathfrak{M}_{256}(2,1,4)$ & $\overline{D_{8}}$ & $\mathfrak{M}_{256}(2,1,1)$ & $S_{3}$ \\
\hline $\mathfrak{M}_{256}(1,2,5: 1)$ & $S_{3}$ & $\mathfrak{M}_{256}(1,2,5: 2)$ & 2 \\
\hline $\mathfrak{M}_{256}(0,2,1: 1)$ & $2^{2}$ & $\mathfrak{M}_{256}(0,2,1: 2)$ & 2 \\
\hline $\mathfrak{M}_{256}(1,1,2: 1)$ & $2^{2}$ & $\mathfrak{M}_{256}(1,1,2: 2)$ & 2 \\
\hline $\mathfrak{M}_{256}(2,1,2: 1)$ & $2^{2}$ & $\mathfrak{M}_{256}(2,1,2: 2)$ & 2 \\
\hline $\mathfrak{M}_{256}(2,4,1: 1)$ & $2^{2}$ & $\mathfrak{M}_{256}(2,4,1: 2)$ & 2 \\
\hline $\mathfrak{M}_{256}(1,1,1)$ & $2^{2}$ & $\mathfrak{M}_{256}(0,1,1)$ & $2^{2}$ \\
\hline $\mathfrak{M}_{256}(2,1,3)$ & 4 & $\mathfrak{M}_{256}(0,1,3)$ & 4 \\
\hline $\mathfrak{M}_{256}(1,1,5)$ & 3 & $\mathfrak{M}_{256}(2,2,1)$ & 2 \\
\hline $\mathfrak{M}_{256}(1,2,1: 1)$ & 2 & $\mathfrak{M}_{256}(1,2,1: 2)$ & 1 \\
\hline $\mathfrak{M}_{256}(1,2,2: 1)$ & 2 & $\mathfrak{M}_{256}(1,2,2: 2)$ & 1 \\
\hline $\mathfrak{M}_{256}(0,1,2)$ & 2 & $\mathfrak{M}_{256}(0,2,2: 1)$ & 2 \\
\hline $\mathfrak{M}_{256}(0,2,2: 2)$ & 1 & $\mathfrak{M}_{256}(2,2,3: 1)$ & 2 \\
\hline $\mathfrak{M}_{256}(2,2,3: 2)$ & 1 & $\mathfrak{M}_{256}(0,2,4: 1)$ & 2 \\
\hline $\mathfrak{M}_{256}(0,2,4: 2)$ & 1 & $\mathfrak{M}_{256}(1,2,4: 1)$ & 2 \\
\hline $\mathfrak{M}_{256}(1,2,4: 2)$ & 1 & $\mathfrak{M}_{256}(0,2,5)$ & 2 \\
\hline $\mathfrak{M}_{256}(1,1,3)$ & 2 & $\mathfrak{M}_{256}(2,4,3)$ & 2 \\
\hline $\mathfrak{M}_{256}(1,1,4)$ & 2 & $\mathfrak{M}_{256}(2,2,4)$ & 2 \\
\hline $\mathfrak{M}_{256}(2,4,4)$ & 2 & $\mathfrak{M}_{256}(2,2,5)$ & 2 \\
\hline $\mathfrak{M}_{256}(0,4,5)$ & 2 & $\mathfrak{M}_{256}(0,4,2: 1)$ & 2 \\
\hline $\mathfrak{M}_{256}(0,4,2: 2)$ & 1 & $\mathfrak{M}_{256}(0,2,3)$ & 1 \\
\hline $\mathfrak{M}_{256}(2,2,2)$ & 1 & $\mathfrak{M}_{256}(1,2,3)$ & 1 \\
\hline $\mathfrak{M}_{266}(2,1,5)$ & $D_{12}$ & $\mathfrak{M}_{266}(1,1,3)$ & $2^{3}$ \\
\hline $\mathfrak{M}_{266}(1,1,2)$ & $S_{3}$ & $\mathfrak{M}_{266}(4,2,5)$ & $S_{3}$ \\
\hline $\mathfrak{M}_{266}(1,1,1)$ & $2^{2}$ & $\mathfrak{M}_{266}(2,3,1)$ & $2^{2}$ \\
\hline $\mathfrak{M}_{266}(2,1,3)$ & $2^{2}$ & $\mathfrak{M}_{266}(1,2,2)$ & $2^{2}$ \\
\hline $\mathfrak{M}_{266}(1,4,2)$ & $2^{2}$ & $\mathfrak{M}_{266}(2,1,4)$ & $2^{2}$ \\
\hline $\mathfrak{M}_{266}(2,2,5)$ & $2^{2}$ & $\mathfrak{M}_{266}(2,2,3)$ & 2 \\
\hline $\mathfrak{M}_{266}(2,4,3)$ & 2 & $\mathfrak{M}_{266}(1,2,1)$ & 2 \\
\hline $\mathfrak{M}_{266}(1,4,1)$ & 2 & $\mathfrak{M}_{266}(2,2,2: 1)$ & 2 \\
\hline $\mathfrak{M}_{266}(2,2,2: 2)$ & 1 & $\mathfrak{M}_{266}(2,2,4)$ & 2 \\
\hline $\mathfrak{M}_{266}(4,2,2)$ & 2 & $\mathfrak{M}_{266}(2,2,1)$ & 1 \\
\hline $\mathfrak{M}_{266}(2,1,1)$ & 1 & $\mathfrak{M}_{266}(4,2,1)$ & 1 \\
\hline$* * * * * * * * * * * *$ & $* * * * *$ & $* * * * * * * * * * * *$ & $* * * * *$ \\
\hline $\mathfrak{M}_{333}(3,3,1: 1)$ & $3^{2} .2$ & $\mathfrak{M}_{333}(3,1,3: 2)$ & $D_{8}$ \\
\hline
\end{tabular}




\begin{tabular}{|c|c|c|c|}
\hline $\mathfrak{M}_{333}(1,1,1)$ & $D_{10}$ & $\mathfrak{M}_{333}(3,3,3: 1)$ & $2^{3}$ \\
\hline $\mathfrak{M}_{333}(3,3,3: 2)$ & $S_{3}$ & $\mathfrak{M}_{333}(3,1,1)$ & $S_{3}$ \\
\hline$\overline{\mathfrak{M}}_{334}(3,0,0)$ & $\overline{2^{4} . S_{3}}$ & $\mathfrak{M}_{334}(1,0,4)$ & $\overline{\overline{S_{4}}}$ \\
\hline $\mathfrak{M}_{334}(1,0,2)$ & $S_{4}$ & $\mathfrak{M}_{334}(3,4,4: 1)$ & $3^{2} .2$ \\
\hline $\mathfrak{M}_{334}(3,4,4: 2)$ & $2^{3}$ & $\mathfrak{M}_{334}(1,4,4)$ & $3^{2} .2$ \\
\hline $\mathfrak{M}_{334}(3,0,2)$ & $D_{12}$ & $\mathfrak{M}_{334}(1,2,2: 1)$ & $D_{10}$ \\
\hline $\mathfrak{M}_{334}(1,2,2: 2)$ & 2 & $\mathfrak{M}_{334}(3,2,4: 1)$ & $D_{8}$ \\
\hline $\mathfrak{M}_{334}(3,2,4: 2)$ & $S_{3}$ & $\mathfrak{M}_{334}(1,2,4: 1)$ & $D_{8}$ \\
\hline $\mathfrak{M}_{334}(1,2,4: 2)$ & $S_{3}$ & $\mathfrak{M}_{334}(3,2,2: 1)$ & $2^{2}$ \\
\hline $\mathfrak{M}_{334}(3,2,2: 2)$ & 2 & & \\
\hline$\overline{\mathfrak{M}_{335}(3,2,6)}$ & $\overline{S_{4}}$ & $\bar{M}_{335}(3,6,6)$ & $3^{2} .2$ \\
\hline $\mathfrak{M}_{335}(1,2,6)$ & $D_{10}$ & $\mathfrak{M}_{335}(3,4,6: 1)$ & $D_{8}$ \\
\hline $\mathfrak{M}_{335}(3,4,6: 2)$ & $S_{3}$ & $\mathfrak{M}_{335}(3,2,4: 1)$ & $D_{8}$ \\
\hline $\mathfrak{M}_{335}(3,2,4: 2)$ & 2 & $\mathfrak{M}_{335}(1,2,2)$ & $D_{8}$ \\
\hline $\mathfrak{M}_{335}(1,4,6)$ & $S_{3}$ & $\mathfrak{M}_{335}(3,4,4: 1)$ & $S_{3}$ \\
\hline $\mathfrak{M}_{335}(3,4,4: 2)$ & $2^{2}$ & $\mathfrak{M}_{335}(3,4,4: 3)$ & 2 \\
\hline $\mathfrak{M}_{335}(3,4,4: 4)$ & 1 & $\mathfrak{M}_{335}(1,2,4: 1)$ & $S_{3}$ \\
\hline $\mathfrak{M}_{335}(1,2,4: 2)$ & 2 & $\mathfrak{M}_{335}(3,2,2: 1)$ & $S_{3}$ \\
\hline $\mathfrak{M}_{335}(3,2,2: 2)$ & $2^{2}$ & $\mathfrak{M}_{335}(1,4,4)$ & 2 \\
\hline$\overline{\mathfrak{M}_{336}(3,4,1)}$ & 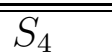 & $\overline{\mathfrak{M}_{336}(1,3,1)}$ & $\overline{33^{2} .2}$ \\
\hline $\mathfrak{M}_{336}(3,2,1)$ & $2^{3} .2$ & $\mathfrak{M}_{336}(1,4,4)$ & $D_{10}$ \\
\hline $\mathfrak{M}_{336}(1,3,3)$ & $S_{3}$ & $\mathfrak{M}_{336}(3,3,2: 1)$ & $S_{3}$ \\
\hline $\mathfrak{M}_{336}(3,3,2: 2)$ & $2^{2}$ & $\mathfrak{M}_{336}(3,3,2: 3)$ & 2 \\
\hline $\mathfrak{M}_{336}(1,4,3: 1)$ & $S_{3}$ & $\mathfrak{M}_{336}(1,4,3: 2)$ & 4 \\
\hline $\mathfrak{M}_{336}(3,4,4)$ & $S_{3}$ & $\mathfrak{M}_{336}(1,3,2: 1)$ & $2^{2}$ \\
\hline $\mathfrak{M}_{336}(1,3,2: 2)$ & 2 & $\mathfrak{M}_{336}(3,4,3)$ & $2^{2}$ \\
\hline $\mathfrak{M}_{336}(1,2,2)$ & 2 & $\mathfrak{M}_{336}(3,4,2)$ & 2 \\
\hline $\mathfrak{M}_{336}(3,3,3)$ & 2 & & \\
\hline $\mathfrak{M}_{344}(4,0,0)$ & $2^{4} . S_{3}$ & $\mathfrak{M}_{344}(0,0,4)$ & $22^{4} .3$ \\
\hline $\mathfrak{M}_{344}(2,4,0)$ & $S_{4}$ & $\mathfrak{M}_{344}(4,4,2: 1)$ & $3^{2} .2$ \\
\hline $\mathfrak{M}_{344}(4,4,2: 2)$ & $D_{8}$ & $\mathfrak{M}_{344}(2,2,0)$ & $D_{12}$ \\
\hline $\mathfrak{M}_{344}(0,4,2)$ & $D_{12}$ & $\mathfrak{M}_{344}(4,4,4: 1)$ & $2^{3}$ \\
\hline $\mathfrak{M}_{344}(4,4,4: 2)$ & $S_{3}$ & $\mathfrak{M}_{344}(2,4,2: 1)$ & $D_{8}$ \\
\hline $\mathfrak{M}_{344}(4,2,2: 2)$ & 2 & $\mathfrak{M}_{344}(0,2,2)$ & $D_{8}$ \\
\hline $\mathfrak{M}_{344}(2,0,4)$ & $D_{8}$ & $\mathfrak{M}_{344}(2,4,4: 1)$ & $S_{3}$ \\
\hline $\mathfrak{M}_{344}(2,4,4: 2)$ & $2^{2}$ & $\mathfrak{M}_{344}(2,2,4: 1)$ & $2^{2}$ \\
\hline $\mathfrak{M}_{344}(2,2,4: 2)$ & 3 & $\mathfrak{M}_{344}(2,2,4: 3)$ & 2 \\
\hline $\mathfrak{M}_{344}(2,2,2)$ & 2 & & \\
\hline$\overline{\mathfrak{M}}_{345}(0,6,2)$ & $S_{4}$ & $\mathfrak{M}_{345}(4,6,6: 1)$ & $3^{2} .2$ \\
\hline $\mathfrak{M}_{345}(4,6,6: 2)$ & $D_{8}$ & $\mathfrak{M}_{345}(0,4,2)$ & $D_{12}$ \\
\hline $\mathfrak{M}_{345}(4,2,6)$ & $D_{12}$ & $\mathfrak{M}_{345}(0,4,6)$ & $D_{12}$ \\
\hline $\mathfrak{M}_{345}(2,6,2)$ & $D_{10}$ & $\mathfrak{M}_{345}(4,4,2: 1)$ & $D_{8}$ \\
\hline
\end{tabular}




\begin{tabular}{|c|c|c|c|}
\hline $\mathfrak{M}_{345}(4,4,2: 2)$ & $S_{3}$ & $\mathfrak{M}_{345}(0,4,4: 1)$ & $D_{8}$ \\
\hline $\mathfrak{M}_{345}(0,4,4: 2)$ & 3 & $\mathfrak{M}_{345}(4,4,6: 1)$ & $D_{8}$ \\
\hline $\mathfrak{M}_{345}(4,4,6: 2)$ & 2 & $\mathfrak{M}_{345}(2,6,6)$ & $\overline{D_{8}}$ \\
\hline $\mathfrak{M}_{345}(0,2,6)$ & $D_{8}$ & $\mathfrak{M}_{345}(4,2,2: 1)$ & $D_{8}$ \\
\hline $\mathfrak{M}_{345}(4,2,2: 2)$ & $S_{3}$ & $\mathfrak{M}_{345}(0,2,4)$ & $D_{8}$ \\
\hline $\mathfrak{M}_{345}(2,6,4: 1)$ & $S_{3}$ & $\mathfrak{M}_{345}(2,6,4: 2)$ & 2 \\
\hline $\mathfrak{M}_{345}(4,4,4: 1)$ & $S_{3}$ & $\mathfrak{M}_{345}(4,4,4: 2)$ & $2^{2}$ \\
\hline $\mathfrak{M}_{345}(4,4,4: 3)$ & 2 & $\mathfrak{M}_{345}(4,4,4: 4)$ & 1 \\
\hline $\mathfrak{M}_{345}(4,6,4)$ & $S_{3}$ & & \\
\hline $\mathfrak{M}_{345}(4,2,4: 1)$ & $2^{2}$ & $\mathfrak{M}_{345}(4,2,4: 2)$ & 2 \\
\hline $\mathfrak{M}_{345}(2,2,2)$ & $2^{2}$ & $\mathfrak{M}_{345}(2,2,4: 1)$ & 3 \\
\hline $\mathfrak{M}_{345}(2,2,4: 2)$ & 2 & $\mathfrak{M}_{345}(2,4,6)$ & 2 \\
\hline $\mathfrak{M}_{345}(2,4,2)$ & 2 & $\mathfrak{M}_{345}(2,4,4: 1)$ & 2 \\
\hline $\mathfrak{M}_{345}(2,4,4,: 2)$ & 1 & $\mathfrak{M}_{345}(2,2,6)$ & 2 \\
\hline 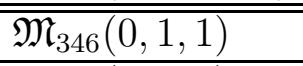 & $\overline{2^{4} . S_{3}}$ & 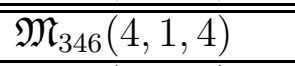 & $\overline{\left(S_{3} \times S_{4}\right)^{+}}$ \\
\hline $\mathfrak{M}_{346}(0,3,4)$ & $S_{4}$ & $\mathfrak{M}_{346}(4,3,1)$ & $3^{2} .2$ \\
\hline $\mathfrak{M}_{346}(4,2,1)$ & $2^{3} .2$ & $\mathfrak{M}_{346}(4,1,5)$ & $2^{3} .2$ \\
\hline $\mathfrak{M}_{346}(2,4,1)$ & $D_{12}$ & $\mathfrak{M}_{346}(2,1,2)$ & $D_{12}$ \\
\hline $\mathfrak{M}_{346}(4,2,4)$ & $D_{12}$ & $\mathfrak{M}_{346}(4,4,4)$ & $D_{12}$ \\
\hline $\mathfrak{M}_{346}(2,3,1)$ & $D_{12}$ & $\mathfrak{M}_{346}(0,4,2)$ & $D_{8}$ \\
\hline $\mathfrak{M}_{346}(2,2,1)$ & $D_{8}$ & $\mathfrak{M}_{346}(4,4,2)$ & $D_{8}$ \\
\hline $\mathfrak{M}_{346}(4,2,2: 1)$ & $2^{3}$ & $\mathfrak{M}_{346}(0,2,2)$ & $2^{3}$ \\
\hline $\mathfrak{M}_{346}(4,2,2: 2)$ & 2 & & \\
\hline $\mathfrak{M}_{346}(2,3,2: 1)$ & $\overline{S_{3}}$ & $\mathfrak{M}_{346}(2,3,2: 2)$ & 2 \\
\hline $\mathfrak{M}_{346}(2,1,3)$ & $S_{3}$ & $\mathfrak{M}_{346}(0,3,3)$ & $S_{3}$ \\
\hline $\mathfrak{M}_{346}(0,3,5)$ & $S_{3}$ & $\mathfrak{M}_{346}(4,4,5)$ & $S_{3}$ \\
\hline $\mathfrak{M}_{346}(4,3,2: 1)$ & $S_{3}$ & $\mathfrak{M}_{346}(4,3,2: 2)$ & 2 \\
\hline $\mathfrak{M}_{346}(2,3,4)$ & $S_{3}$ & $\mathfrak{M}_{346}(2,4,2: 1)$ & $2^{2}$ \\
\hline $\mathfrak{M}_{346}(2,4,2: 2)$ & 2 & $\mathfrak{M}_{346}(2,2,2: 1)$ & $2^{2}$ \\
\hline $\mathfrak{M}_{346}(2,2,2: 2)$ & 2 & $\mathfrak{M}_{346}(2,2,4)$ & $2^{2}$ \\
\hline $\mathfrak{M}_{346}(4,3,5)$ & $2^{2}$ & $\mathfrak{M}_{346}(2,3,5: 1)$ & $2^{2}$ \\
\hline $\mathfrak{M}_{346}(2,3,5: 2)$ & 2 & & \\
\hline $\mathfrak{M}_{346}(0,2,3)$ & 3 & $\mathfrak{M}_{346}(2,4,3: 1)$ & 3 \\
\hline $\mathfrak{M}_{346}(4,3,3)$ & 2 & $\mathfrak{M}_{346}(2,4,3: 2)$ & 2 \\
\hline $\mathfrak{M}_{346}(2,4,5)$ & 2 & $\mathfrak{M}_{346}(2,3,3: 1)$ & 2 \\
\hline $\mathfrak{M}_{346}(2,3,3: 2)$ & 1 & $\mathfrak{M}_{346}(4,4,3)$ & 2 \\
\hline $\mathfrak{M}_{346}(4,2,5)$ & 2 & $\mathfrak{M}_{346}(2,2,3)$ & 1 \\
\hline $\mathfrak{M}_{355}(2,2,4)$ & $D_{12}$ & $\mathfrak{M}_{355}(2,6,4: 1)$ & $D_{8}$ \\
\hline $\mathfrak{M}_{355}(2,6,4: 2)$ & $S_{3}$ & $\mathfrak{M}_{355}(6,4,4)$ & $S_{3}$ \\
\hline $\mathfrak{M}_{355}(4,6,8: 1)$ & $S_{3}$ & $\mathfrak{M}_{355}(4,6,8: 2)$ & 2 \\
\hline $\mathfrak{M}_{355}(6,6,8)$ & $S_{3}$ & $\mathfrak{M}_{355}(2,2,8: 1)$ & $2^{2}$ \\
\hline $\mathfrak{M}_{355}(2,2,8: 2)$ & 3 & $\mathfrak{M}_{355}(2,2,8: 3)$ & 2 \\
\hline $\mathfrak{M}_{355}(4,2,8)$ & 2 & $\mathfrak{M}_{355}(4,4,8: 1)$ & 2 \\
\hline
\end{tabular}




\begin{tabular}{|c|c|c|c|}
\hline $\mathfrak{M}_{355}(4,4,8: 2)$ & 1 & $\mathfrak{M}_{355}(2,6,6)$ & 2 \\
\hline $\mathfrak{M}_{355}(4,4,4: 1)$ & 2 & $\mathfrak{M}_{355}(4,4,4: 2)$ & 1 \\
\hline $\mathfrak{M}_{355}(2,4,6: 1)$ & 2 & $\mathfrak{M}_{355}(4,2,6: 2)$ & 1 \\
\hline $\mathfrak{M}_{355}(4,6,6)$ & 2 & $\mathfrak{M}_{355}(2,2,6)$ & 2 \\
\hline $\mathfrak{M}_{355}(2,4,4)$ & 2 & $\mathfrak{M}_{355}(4,4,6: 1)$ & 2 \\
\hline $\mathfrak{M}_{355}(4,4,6: 2)$ & 1 & & \\
\hline$\overline{\mathfrak{M}_{356}(6,1,1)}$ & $\overline{3^{2} .2}$ & $\overline{\bar{M}_{356}(4,1,1)}$ & $\overline{D_{12}}$ \\
\hline $\mathfrak{M}_{356}(2,1,5)$ & $D_{12}$ & $\mathfrak{M}_{356}(2,2,5: 1)$ & $D_{8}$ \\
\hline $\mathfrak{M}_{356}(2,2,5: 2)$ & 2 & $\mathfrak{M}_{356}(4,1,4)$ & $D_{8}$ \\
\hline $\mathfrak{M}_{356}(2,3,1: 1)$ & $S_{3}$ & $\mathfrak{M}_{356}(2,3,1: 2)$ & $2^{2}$ \\
\hline $\mathfrak{M}_{356}(2,3,1: 3)$ & 2 & $\mathfrak{M}_{356}(2,1,2)$ & $S_{3}$ \\
\hline $\mathfrak{M}_{356}(6,4,2)$ & $S_{3}$ & $\mathfrak{M}_{356}(4,3,1: 1)$ & $S_{3}$ \\
\hline $\mathfrak{M}_{356}(4,3,1: 2)$ & 2 & $\mathfrak{M}_{356}(2,3,5)$ & $S_{3}$ \\
\hline $\mathfrak{M}_{356}(6,4,1)$ & $2^{2}$ & $\mathfrak{M}_{356}(6,2,1)$ & $2^{2}$ \\
\hline $\mathfrak{M}_{356}(2,3,2: 1)$ & $2^{2}$ & $\mathfrak{M}_{356}(2,3,2: 2)$ & 2 \\
\hline $\mathfrak{M}_{356}(4,1,2)$ & $2^{2}$ & $\mathfrak{M}_{356}(4,1,3)$ & 4 \\
\hline $\mathfrak{M}_{356}(6,3,3)$ & 4 & $\mathfrak{M}_{356}(2,4,4)$ & 3 \\
\hline $\mathfrak{M}_{356}(2,2,1)$ & 2 & $\mathfrak{M}_{356}(4,4,1)$ & 2 \\
\hline $\mathfrak{M}_{356}(2,2,2: 1)$ & 2 & $\mathfrak{M}_{356}(2,2,2: 2)$ & 1 \\
\hline $\mathfrak{M}_{356}(4,3,2: 1)$ & 2 & $\mathfrak{M}_{356}(4,3,2: 2)$ & 1 \\
\hline $\mathfrak{M}_{356}(6,3,2)$ & 2 & $\mathfrak{M}_{356}(4,3,3: 1)$ & 2 \\
\hline $\mathfrak{M}_{356}(4,3,3: 2)$ & 1 & $\mathfrak{M}_{356}(2,4,3)$ & 2 \\
\hline $\mathfrak{M}_{356}(6,2,4)$ & 2 & $\mathfrak{M}_{356}(2,3,4)$ & 2 \\
\hline $\mathfrak{M}_{356}(4,3,5)$ & 2 & $\mathfrak{M}_{356}(4,2,5: 1)$ & 2 \\
\hline $\mathfrak{M}_{356}(4,2,5: 2)$ & 1 & $\mathfrak{M}_{356}(4,4,5)$ & 2 \\
\hline $\mathfrak{M}_{356}(6,2,3)$ & 2 & $\mathfrak{M}_{356}(4,2,3)$ & 2 \\
\hline $\mathfrak{M}_{356}(4,2,4: 1)$ & 2 & $\mathfrak{M}_{356}(4,2,4: 2)$ & 1 \\
\hline $\mathfrak{M}_{356}(2,2,4)$ & 2 & $\mathfrak{M}_{356}(2,4,5)$ & 2 \\
\hline $\mathfrak{M}_{356}(4,2,1: 1)$ & 2 & $\mathfrak{M}_{356}(4,2,1: 2)$ & 1 \\
\hline $\mathfrak{M}_{356}(4,2,2)$ & 1 & $\mathfrak{M}_{356}(4,4,2)$ & 1 \\
\hline $\mathfrak{M}_{356}(2,3,3)$ & 1 & $\mathfrak{M}_{356}(4,3,4)$ & 1 \\
\hline $\mathfrak{M}_{356}(4,4,4)$ & 1 & & \\
\hline $\mathfrak{M}_{366}(4,2,5)$ & $\overline{D_{8}}$ & 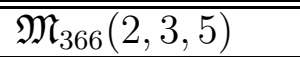 & $S_{3}$ \\
\hline $\mathfrak{M}_{366}(4,4,3)$ & $2^{2}$ & & \\
\hline $\mathfrak{M}_{366}(3,1,1)$ & $2^{2}$ & $\mathfrak{M}_{366}(2,2,2: 1)$ & $2^{2}$ \\
\hline $\mathfrak{M}_{366}(2,2,2: 2)$ & 1 & $\mathfrak{M}_{366}(3,3,2)$ & $2^{2}$ \\
\hline $\mathfrak{M}_{366}(2,4,4)$ & $2^{2}$ & $\mathfrak{M}_{366}(4,4,2)$ & $2^{2}$ \\
\hline $\mathfrak{M}_{366}(3,2,3)$ & 2 & $\mathfrak{M}_{366}(3,3,3)$ & 2 \\
\hline $\mathfrak{M}_{366}(3,4,1: 1)$ & 2 & $\mathfrak{M}_{366}(3,4,1: 2)$ & 1 \\
\hline $\mathfrak{M}_{366}(3,3,1: 1)$ & 2 & $\mathfrak{M}_{366}(3,3,1: 2)$ & 1 \\
\hline $\mathfrak{M}_{366}(4,4,1)$ & 2 & $\mathfrak{M}_{366}(2,4,2)$ & 2 \\
\hline $\mathfrak{M}_{366}(3,3,4)$ & 2 & $\mathfrak{M}_{366}(3,4,2)$ & 2 \\
\hline
\end{tabular}




\begin{tabular}{|c|c|c|c|}
\hline $\mathfrak{M}_{366}(2,3,2)$ & 2 & $\mathfrak{M}_{366}(3,2,1)$ & 1 \\
\hline $\mathfrak{M}_{366}(2,4,1)$ & 1 & $\mathfrak{M}_{366}(2,2,1)$ & 1 \\
\hline$* * * * * * * * * * * *$ & $* * * * *$ & $* * * * * * * * * * * *$ & $* * * * *$ \\
\hline $\mathfrak{M}_{444}(2,2,0)$ & $D_{12}$ & $\mathfrak{M}_{444}(2,4,0)$ & $D_{8}$ \\
\hline $\mathfrak{M}_{444}(2,2,4: 1)$ & $S_{3}$ & $\mathfrak{M}_{444}(2,2,4: 2)$ & $2^{2}$ \\
\hline $\mathfrak{M}_{444}(2,2,4: 3)$ & 1 & $\mathfrak{M}_{444}(4,2,4: 1)$ & $2^{2}$ \\
\hline $\mathfrak{M}_{444}(4,2,4: 2)$ & 2 & $\mathfrak{M}_{444}(4,4,4)$ & 2 \\
\hline $\mathfrak{M}_{444}(2,2,2)$ & 2 & & \\
\hline$\overline{\mathfrak{M}_{445}(0,6,2)}$ & $\overline{D_{12}}$ & $\mathfrak{M}_{445}(4,6,2)$ & $D_{8}$ \\
\hline $\mathfrak{M}_{445}(2,2,2)$ & $D_{8}$ & $\mathfrak{M}_{445}(0,6,4)$ & $D_{8}$ \\
\hline $\mathfrak{M}_{445}(2,6,4: 1)$ & $S_{3}$ & $\mathfrak{M}_{445}(2,6,4: 2)$ & 1 \\
\hline $\mathfrak{M}_{445}(4,2,2: 1)$ & $S_{3}$ & $\mathfrak{M}_{445}(4,2,2: 2)$ & $2^{2}$ \\
\hline $\mathfrak{M}_{445}(4,6,4: 1)$ & $2^{2}$ & $\mathfrak{M}_{445}(4,6,4: 2)$ & 1 \\
\hline $\mathfrak{M}_{445}(4,6,6: 1)$ & $2^{2}$ & $\mathfrak{M}_{445}(4,6,6: 2)$ & 2 \\
\hline $\mathfrak{M}_{445}(4,4,2: 1)$ & $2^{2}$ & $\mathfrak{M}_{445}(4,4,2: 2)$ & 3 \\
\hline $\mathfrak{M}_{445}(4,4,2: 3)$ & 2 & $\mathfrak{M}_{445}(2,6,6)$ & $2^{2}$ \\
\hline $\mathfrak{M}_{445}(0,4,4)$ & 3 & $\mathfrak{M}_{445}(2,4,2)$ & 2 \\
\hline $\mathfrak{M}_{445}(4,4,4: 1)$ & 2 & $\mathfrak{M}_{445}(4,4,4: 2)$ & 1 \\
\hline$\overline{\mathfrak{M}}_{445}(2,6,2)$ & 2 & $\mathfrak{M}_{445}(2,4,4: 1)$ & 2 \\
\hline $\mathfrak{M}_{445}(2,4,4: 2)$ & 1 & & \\
\hline$\overline{\mathfrak{M}_{446}(0,1,1)}$ & $\overline{2^{3} . S_{4}}$ & $\mathfrak{M}_{446}(2,4,1)$ & $3^{2} .2^{2}$ \\
\hline $\mathfrak{M}_{446}(0,4,2)$ & $S_{4}$ & $\mathfrak{M}_{446}(4,4,4)$ & $S_{4}$ \\
\hline $\mathfrak{M}_{446}(2,1,2)$ & $D_{12}$ & $\mathfrak{M}_{446}(2,1,5)$ & $D_{8}$ \\
\hline $\mathfrak{M}_{446}(4,1,2)$ & $2^{3}$ & $\mathfrak{M}_{446}(0,5,2)$ & $2^{3}$ \\
\hline $\mathfrak{M}_{446}(4,3,1)$ & $S_{3}$ & $\mathfrak{M}_{446}(2,4,3: 1)$ & $S_{3}$ \\
\hline $\mathfrak{M}_{446}(2,4,3: 2)$ & 4 & $\mathfrak{M}_{446}(2,3,1)$ & $2^{2}$ \\
\hline $\mathfrak{M}_{446}(2,4,2)$ & $2^{2}$ & $\mathfrak{M}_{446}(2,5,2: 1)$ & $2^{2}$ \\
\hline $\mathfrak{M}_{446}(2,5,2: 2)$ & 2 & $\mathfrak{M}_{446}(4,4,3)$ & $2^{2}$ \\
\hline $\mathfrak{M}_{446}(4,4,5)$ & $2^{2}$ & $\mathfrak{M}_{446}(4,3,5: 1)$ & 3 \\
\hline $\mathfrak{M}_{446}(4,3,5: 2)$ & 2 & $\mathfrak{M}_{446}(4,3,5: 3)$ & 1 \\
\hline $\mathfrak{M}_{446}(0,3,3)$ & 3 & $\mathfrak{M}_{446}(2,2,2)$ & 2 \\
\hline $\mathfrak{M}_{446}(4,3,2: 1)$ & 2 & $\mathfrak{M}_{446}(4,3,2: 2)$ & 1 \\
\hline $\mathfrak{M}_{446}(4,2,2)$ & 2 & $\mathfrak{M}_{446}(4,5,2)$ & 2 \\
\hline $\mathfrak{M}_{446}(4,5,5)$ & 2 & $\mathfrak{M}_{446}(2,5,5)$ & 2 \\
\hline $\mathfrak{M}_{446}(2,3,2: 1)$ & 2 & $\mathfrak{M}_{446}(2,3,2: 2)$ & 1 \\
\hline $\mathfrak{M}_{446}(2,3,3)$ & 1 & $\mathfrak{M}_{446}(2,3,5)$ & 1 \\
\hline $\mathfrak{M}_{446}(4,3,3)$ & 1 & & \\
\hline$\overline{\mathfrak{M}_{455}(6,6,4)}$ & 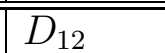 & $\mathfrak{M}_{455}(6,2,8)$ & $D_{8}$ \\
\hline $\mathfrak{M}_{455}(2,6,4: 1)$ & $D_{8}$ & $\mathfrak{M}_{455}(2,6,4: 2)$ & 2 \\
\hline $\mathfrak{M}_{455}(6,6,8: 1)$ & $S_{3}$ & $\mathfrak{M}_{455}(6,6,8: 2)$ & $2^{2}$ \\
\hline $\mathfrak{M}_{455}(6,6,8: 3)$ & 1 & $\mathfrak{M}_{455}(4,2,4: 1)$ & $S_{3}$ \\
\hline $\mathfrak{M}_{455}(2,4,4: 2)$ & 2 & $\mathfrak{M}_{455}(2,2,8: 1)$ & $2^{2}$ \\
\hline
\end{tabular}




\begin{tabular}{|c|c|c|c|}
\hline $\mathfrak{M}_{455}(2,2,8: 2)$ & 2 & $\mathfrak{M}_{455}(4,4,8: 1)$ & 2 \\
\hline $\mathfrak{M}_{455}(4,4,8: 2)$ & 1 & $\mathfrak{M}_{455}(6,2,6)$ & 2 \\
\hline $\mathfrak{M}_{455}(6,6,6)$ & 2 & $\mathfrak{M}_{455}(2,2,6)$ & 2 \\
\hline $\mathfrak{M}_{455}(4,6,4)$ & 2 & $\mathfrak{M}_{455}(4,2,8)$ & 2 \\
\hline $\mathfrak{M}_{455}(4,4,4: 1)$ & 2 & $\mathfrak{M}_{455}(4,4,4: 2)$ & 1 \\
\hline $\mathfrak{M}_{455}(6,4,8)$ & 1 & $\mathfrak{M}_{455}(4,6,6)$ & 1 \\
\hline $\mathfrak{M}_{455}(2,4,6)$ & 1 & $\mathfrak{M}_{455}(4,4,6)$ & 1 \\
\hline$\overline{\mathfrak{M}_{456}(2,1,1)}$ & $\overline{D_{12}}$ & 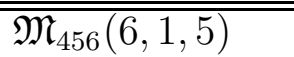 & $\overline{\overline{D_{12}}}$ \\
\hline $\mathfrak{M}_{456}(6,1,4)$ & $D_{8}$ & $\mathfrak{M}_{456}(2,1,4)$ & $D_{8}$ \\
\hline $\mathfrak{M}_{456}(2,5,5: 1)$ & $D_{8}$ & $\mathfrak{M}_{456}(4,1,5)$ & $D_{8}$ \\
\hline $\mathfrak{M}_{456}(4,4,1)$ & $S_{3}$ & $\mathfrak{M}_{456}(4,1,1)$ & $S_{3}$ \\
\hline $\mathfrak{M}_{456}(2,2,1: 1)$ & $S_{3}$ & $\mathfrak{M}_{456}(2,2,1: 2)$ & $2^{2}$ \\
\hline $\mathfrak{M}_{456}(2,2,1: 3)$ & 2 & $\mathfrak{M}_{456}(6,4,4)$ & $\overline{S_{3}}$ \\
\hline $\mathfrak{M}_{456}(2,4,5)$ & $S_{3}$ & $\mathfrak{M}_{456}(4,4,5)$ & $S_{3}$ \\
\hline $\mathfrak{M}_{456}(6,4,1)$ & $2^{2}$ & $\mathfrak{M}_{456}(2,3,1: 1)$ & $2^{2}$ \\
\hline $\mathfrak{M}_{456}(2,3,1: 2)$ & 2 & $\mathfrak{M}_{456}(6,2,1)$ & $2^{2}$ \\
\hline $\mathfrak{M}_{456}(6,5,1: 1)$ & $2^{2}$ & $\mathfrak{M}_{456}(6,5,1: 2)$ & 2 \\
\hline $\mathfrak{M}_{456}(4,2,1: 1)$ & $2^{2}$ & $\mathfrak{M}_{456}(4,2,1: 2)$ & 1 \\
\hline $\mathfrak{M}_{456}(6,2,2: 1)$ & $2^{2}$ & $\mathfrak{M}_{456}(6,2,2: 2)$ & 2 \\
\hline $\mathfrak{M}_{456}(6,2,2: 3)$ & 1 & $\mathfrak{M}_{456}(2,2,2: 1)$ & $2^{2}$ \\
\hline $\mathfrak{M}_{456}(2,2,2: 2)$ & 2 & $\mathfrak{M}_{456}(2,4,2)$ & $2^{2}$ \\
\hline $\mathfrak{M}_{456}(6,1,2)$ & $2^{2}$ & $\mathfrak{M}_{456}(6,4,3)$ & 4 \\
\hline $\mathfrak{M}_{456}(4,4,3: 1)$ & 4 & $\mathfrak{M}_{456}(4,4,3: 2)$ & 2 \\
\hline $\mathfrak{M}_{456}(2,2,4)$ & $2^{2}$ & $\mathfrak{M}_{456}(6,2,4)$ & $2^{2}$ \\
\hline $\mathfrak{M}_{456}(4,3,1: 1)$ & 2 & $\mathfrak{M}_{456}(4,3,1: 2)$ & 1 \\
\hline $\mathfrak{M}_{456}(2,3,2: 1)$ & 2 & $\mathfrak{M}_{456}(2,3,2: 2)$ & 1 \\
\hline $\mathfrak{M}_{456}(4,1,2)$ & 2 & $\mathfrak{M}_{456}(2,3,3)$ & 2 \\
\hline $\mathfrak{M}_{456}(4,1,3)$ & 2 & $\mathfrak{M}_{456}(6,2,3)$ & 2 \\
\hline $\mathfrak{M}_{456}(2,2,3)$ & 2 & $\mathfrak{M}_{456}(2,5,3)$ & 2 \\
\hline $\mathfrak{M}_{456}(4,5,3)$ & 2 & $\mathfrak{M}_{456}(6,3,3: 1)$ & 2 \\
\hline $\mathfrak{M}_{456}(6,3,3: 2)$ & 1 & $\mathfrak{M}_{456}(6,5,4)$ & 2 \\
\hline $\mathfrak{M}_{456}(2,5,4)$ & 2 & $\mathfrak{M}_{456}(4,4,4)$ & 2 \\
\hline $\mathfrak{M}_{456}(2,3,5)$ & 2 & $\mathfrak{M}_{456}(6,2,3)$ & 2 \\
\hline $\mathfrak{M}_{456}(4,5,5: 1)$ & 2 & $\mathfrak{M}_{456}(4,5,5: 2)$ & 1 \\
\hline $\mathfrak{M}_{456}(6,3,1)$ & 2 & & \\
\hline $\mathfrak{M}_{456}(4,5,1: 1)$ & 2 & $\mathfrak{M}_{456}(4,5,1: 2)$ & 1 \\
\hline $\mathfrak{M}_{456}(4,4,2)$ & 2 & $\mathfrak{M}_{456}(2,5,5: 2)$ & 2 \\
\hline $\mathfrak{M}_{456}(4,2,5)$ & 2 & $\mathfrak{M}_{456}(6,2,5)$ & 2 \\
\hline $\mathfrak{M}_{456}(4,2,2)$ & 1 & $\mathfrak{M}_{456}(4,5,2)$ & 1 \\
\hline $\mathfrak{M}_{456}(6,5,2)$ & 1 & $\mathfrak{M}_{456}(4,3,2)$ & 1 \\
\hline $\mathfrak{M}_{456}(6,3,2)$ & 1 & $\mathfrak{M}_{456}(4,2,3)$ & 1 \\
\hline $\mathfrak{M}_{456}(6,3,4)$ & 1 & $\mathfrak{M}_{456}(4,3,4)$ & 1 \\
\hline
\end{tabular}




\begin{tabular}{|c|c|c|c|}
\hline $\mathfrak{M}_{456}(4,2,4)$ & 1 & $\mathfrak{M}_{456}(4,5,4)$ & 1 \\
\hline $\mathfrak{M}_{456}(4,3,5)$ & 1 & & \\
\hline$\overline{\mathfrak{M}_{466}(1,4,5)}$ & $\overline{\left(S_{3} \times S_{4}\right)^{+}}$ & $\overline{\mathfrak{M}_{466}(1,1,3)}$ & $\overline{D_{8} .2^{2}}$ \\
\hline $\mathfrak{M}_{466}(4,4,4)$ & $S_{4}$ & $\mathfrak{M}_{466}(4,5,3)$ & $D_{8}$ \\
\hline $\mathfrak{M}_{466}(2,4,3)$ & $D_{8}$ & $\mathfrak{M}_{466}(5,5,5)$ & $2^{3}$ \\
\hline $\mathfrak{M}_{466}(1,3,2)$ & $S_{3}$ & $\mathfrak{M}_{466}(2,5,5)$ & $S_{3}$ \\
\hline $\mathfrak{M}_{466}(4,2,2)$ & $S_{3}$ & $\mathfrak{M}_{466}(2,2,3)$ & $2^{2}$ \\
\hline $\mathfrak{M}_{466}(2,1,1)$ & $2^{2}$ & $\mathfrak{M}_{466}(4,5,1)$ & $2^{2}$ \\
\hline $\mathfrak{M}_{466}(3,4,2)$ & $2^{2}$ & $\mathfrak{M}_{466}(2,2,2)$ & $2^{2}$ \\
\hline $\mathfrak{M}_{466}(3,5,4)$ & 3 & $\mathfrak{M}_{466}(2,4,1)$ & 2 \\
\hline $\mathfrak{M}_{466}(2,2,1)$ & 2 & $\mathfrak{M}_{466}(3,4,1)$ & 2 \\
\hline $\mathfrak{M}_{466}(1,3,1)$ & 2 & $\mathfrak{M}_{466}(3,5,2: 1)$ & 2 \\
\hline $\mathfrak{M}_{466}(3,5,2: 2)$ & 1 & $\mathfrak{M}_{466}(3,3,2: 1)$ & 2 \\
\hline $\mathfrak{M}_{466}(3,3,2: 2)$ & 1 & $\mathfrak{M}_{466}(3,2,2: 1)$ & 2 \\
\hline $\mathfrak{M}_{466}(3,2,2: 2)$ & 1 & $\mathfrak{M}_{466}(2,5,2)$ & 2 \\
\hline $\mathfrak{M}_{466}(5,5,2)$ & 2 & $\mathfrak{M}_{466}(2,2,4)$ & 2 \\
\hline $\mathfrak{M}_{466}(3,3,5)$ & 2 & $\mathfrak{M}_{466}(3,3,3)$ & 1 \\
\hline $\mathfrak{M}_{466}(3,2,3)$ & 1 & $\mathfrak{M}_{466}(2,3,1)$ & 1 \\
\hline $\mathfrak{M}_{466}(5,5,1)$ & 1 & $\mathfrak{M}_{466}(5,3,1)$ & 1 \\
\hline $\mathfrak{M}_{466}(5,2,1)$ & 1 & & \\
\hline F************ & $* * * * *$ & $* * * * * * * * * * * *$ & $* * * * *$ \\
\hline $\mathfrak{M}_{555}(4,4,8: 1)$ & $2^{2}$ & $\mathfrak{M}_{555}(4,4,8: 2)$ & 2 \\
\hline $\mathfrak{M}_{555}(4,4,8: 3)$ & 1 & $\mathfrak{M}_{555}(8,4,8)$ & 3 \\
\hline $\mathfrak{M}_{555}(8,8,8: 1)$ & 2 & $\mathfrak{M}_{555}(8,8,8: 2)$ & 1 \\
\hline $\mathfrak{M}_{555}(4,4,6)$ & 2 & $\mathfrak{M}_{555}(6,4,8)$ & 1 \\
\hline $\mathfrak{M}_{555}(8,6,8)$ & 1 & $\mathfrak{M}_{555}(6,6,6)$ & 1 \\
\hline $\mathfrak{M}_{555}(6,4,6)$ & 1 & & \\
\hline$\overline{\mathfrak{M}_{556}(4,5,1: 1)}$ & $\overline{\bar{S} S_{3}}$ & $\overline{\mathfrak{M}}_{556}(4,5,1: 2)$ & 2 \\
\hline $\mathfrak{M}_{556}(8,5,1)$ & $S_{3}$ & $\mathfrak{M}_{556}(4,5,4)$ & $2^{2}$ \\
\hline $\mathfrak{M}_{556}(6,1,1)$ & 2 & $\mathfrak{M}_{556}(6,2,1: 1)$ & 2 \\
\hline $\mathfrak{M}_{556}(6,2,1: 2)$ & 1 & $\mathfrak{M}_{556}(8,1,1: 1)$ & 2 \\
\hline $\mathfrak{M}_{556}(8,1,1: 2)$ & 1 & $\mathfrak{M}_{556}(8,4,1: 1)$ & 2 \\
\hline $\mathfrak{M}_{556}(8,4,1: 2)$ & 1 & $\mathfrak{M}_{556}(8,3,1: 1)$ & 2 \\
\hline $\mathfrak{M}_{556}(8,3,1: 2)$ & 1 & $\mathfrak{M}_{556}(4,4,1)$ & 2 \\
\hline $\mathfrak{M}_{556}(8,2,1: 1)$ & 2 & $\mathfrak{M}_{556}(8,2,1: 2)$ & 1 \\
\hline $\mathfrak{M}_{556}(4,3,1)$ & 2 & $\mathfrak{M}_{556}(6,5,1)$ & 2 \\
\hline $\mathfrak{M}_{556}(4,2,1: 1)$ & 2 & $\mathfrak{M}_{556}(4,2,1: 2)$ & 1 \\
\hline $\mathfrak{M}_{556}(6,5,2: 1)$ & 2 & $\mathfrak{M}_{556}(6,5,2: 2)$ & 1 \\
\hline $\mathfrak{M}_{556}(4,2,2: 1)$ & 2 & $\mathfrak{M}_{556}(4,2,2: 2)$ & 1 \\
\hline $\mathfrak{M}_{556}(8,5,2: 1)$ & 2 & $\mathfrak{M}_{556}(8,5,2: 2)$ & 1 \\
\hline $\mathfrak{M}_{556}(4,3,2)$ & 2 & $\mathfrak{M}_{556}(4,3,3: 1)$ & 2 \\
\hline $\mathfrak{M}_{556}(4,3,3: 2)$ & 1 & $\mathfrak{M}_{556}(6,5,5)$ & 2 \\
\hline
\end{tabular}




\begin{tabular}{|c|c|c|c|}
\hline $\mathfrak{M}_{556}(8,5,5)$ & 2 & $\mathfrak{M}_{556}(6,3,1)$ & 1 \\
\hline $\mathfrak{M}_{556}(6,4,1)$ & 1 & $\mathfrak{M}_{556}(8,4,2)$ & 1 \\
\hline $\mathfrak{M}_{556}(6,3,2)$ & 1 & $\mathfrak{M}_{556}(8,3,2)$ & 1 \\
\hline $\mathfrak{M}_{556}(8,2,2)$ & 1 & $\mathfrak{M}_{556}(6,4,2)$ & 1 \\
\hline $\mathfrak{M}_{556}(4,4,2)$ & 1 & $\mathfrak{M}_{556}(6,4,3)$ & 1 \\
\hline $\mathfrak{M}_{556}(6,5,3)$ & 1 & $\mathfrak{M}_{556}(8,5,3)$ & 1 \\
\hline $\mathfrak{M}_{556}(4,5,3)$ & 1 & $\mathfrak{M}_{556}(4,4,3)$ & 1 \\
\hline $\mathfrak{M}_{556}(6,4,4)$ & 1 & $\mathfrak{M}_{556}(6,5,4)$ & 1 \\
\hline $\mathfrak{M}_{557}(6,1,1)$ & $\overline{1} 1$ & & \\
\hline $\mathfrak{M}_{566}(5,5,2)$ & $\overline{\bar{S} S_{3}}$ & $\bar{P}_{\mathfrak{M}_{566}(2,5,5)}$ & $S_{3}$ \\
\hline & & $\mathfrak{M}_{566}(5,5,3)$ & $2^{2}$ \\
\hline $\mathfrak{M}_{566}(1,1,2)$ & $2^{2}$ & $\mathfrak{M}_{566}(1,1,4: 1)$ & $2^{2}$ \\
\hline $\mathfrak{M}_{566}(1,1,4: 2)$ & 2 & $\mathfrak{M}_{566}(1,2,5)$ & $2^{2}$ \\
\hline $\mathfrak{M}_{566}(4,2,5)$ & $2^{2}$ & $\mathfrak{M}_{566}(3,4,5)$ & 4 \\
\hline $\mathfrak{M}_{566}(3,3,5)$ & 4 & $\mathfrak{M}_{566}(3,4,3)$ & 2 \\
\hline $\mathfrak{M}_{566}(3,3,3)$ & 2 & $\mathfrak{M}_{566}(4,4,3)$ & 2 \\
\hline $\mathfrak{M}_{566}(1,1,3)$ & 2 & $\mathfrak{M}_{566}(1,1,1)$ & 2 \\
\hline $\mathfrak{M}_{566}(1,4,1)$ & 2 & $\mathfrak{M}_{566}(3,2,1: 1)$ & 2 \\
\hline $\mathfrak{M}_{566}(3,2,1: 2)$ & 1 & $\mathfrak{M}_{566}(5,5,1)$ & 2 \\
\hline $\mathfrak{M}_{566}(1,5,2)$ & 2 & $\mathfrak{M}_{566}(4,5,2)$ & 2 \\
\hline $\mathfrak{M}_{566}(1,2,2)$ & 2 & $\mathfrak{M}_{566}(2,5,2)$ & 2 \\
\hline $\mathfrak{M}_{566}(2,2,2: 1)$ & 2 & $\mathfrak{M}_{566}(2,2,2: 2)$ & 1 \\
\hline $\mathfrak{M}_{566}(4,3,2)$ & 2 & $\mathfrak{M}_{566}(5,2,2)$ & 2 \\
\hline $\mathfrak{M}_{566}(4,4,2: 1)$ & 2 & $\mathfrak{M}_{566}(4,4,2: 2)$ & 1 \\
\hline $\mathfrak{M}_{566}(1,4,2)$ & 2 & $\mathfrak{M}_{566}(1,3,3)$ & 2 \\
\hline $\mathfrak{M}_{566}(3,2,4)$ & 2 & $\mathfrak{M}_{566}(5,5,4)$ & 2 \\
\hline $\mathfrak{M}_{566}(2,4,4)$ & 2 & $\mathfrak{M}_{566}(3,2,5)$ & 2 \\
\hline $\mathfrak{M}_{566}(2,2,5)$ & 2 & $\mathfrak{M}_{566}(5,1,1)$ & 1 \\
\hline $\mathfrak{M}_{566}(2,5,3)$ & 1 & $\mathfrak{M}_{566}(2,2,3)$ & 1 \\
\hline $\mathfrak{M}_{566}(1,2,1)$ & 1 & $\mathfrak{M}_{566}(3,4,1)$ & 1 \\
\hline $\mathfrak{M}_{566}(4,2,1)$ & 1 & $\mathfrak{M}_{566}(1,3,2)$ & 1 \\
\hline $\mathfrak{M}_{566}(3,3,1)$ & 1 & $\mathfrak{M}_{566}(1,3,1)$ & 1 \\
\hline $\mathfrak{M}_{566}(4,5,1)$ & 1 & $\mathfrak{M}_{566}(3,5,1)$ & 1 \\
\hline $\mathfrak{M}_{566}(5,2,1)$ & 1 & $\mathfrak{M}_{566}(4,4,1)$ & 1 \\
\hline $\mathfrak{M}_{566}(3,3,2)$ & 1 & $\mathfrak{M}_{566}(4,2,2)$ & 1 \\
\hline $\mathfrak{M}_{566}(2,3,2)$ & 1 & & \\
\hline$* * * * * * * * * * * *$ & $* * * * *$ & $* * * * * * * * * * * *$ & $* * * * *$ \\
\hline $\mathfrak{M}_{666}(5,5,2)$ & $3^{2} .2$ & $\mathfrak{M}_{666}(5,1,3)$ & $2^{2}$ \\
\hline $\mathfrak{M}_{666}(3,2,3)$ & $2^{2}$ & $\mathfrak{M}_{666}(5,1,2)$ & $2^{2}$ \\
\hline $\mathfrak{M}_{666}(4,1,4)$ & 3 & $\mathfrak{M}_{666}(2,1,3)$ & 2 \\
\hline $\mathfrak{M}_{666}(5,1,1)$ & 2 & $\mathfrak{M}_{666}(2,2,1: 1)$ & 2 \\
\hline $\mathfrak{M}_{666}(1,2,2: 2)$ & 1 & $\mathfrak{M}_{666}(4,1,2)$ & 2 \\
\hline
\end{tabular}




\begin{tabular}{|l|l|l|l|l|}
\hline $\mathfrak{M}_{666}(2,1,1)$ & 1 & & $\mathfrak{M}_{666}(1,4,1)$ & 1 \\
\hline
\end{tabular}

\section{REFERENCES}

[1] F. BUEKENHOUT, The basic diagram of a geometry, Lecture Notes, Springer, 893(1981).

[2] F. BUEKENHOUT, M. DEHON, D. LEEMANS, All geometries of the Mathieu group $M_{11}$ based on maximal subgroups, Experimental Math. 5(1996), 101-110.

[3] J. H. CONWAY, R. T. CURTIS, S. P. NORTON, R. A. PARKER and R. A. WILSON, An Atlas of Finite Groups, Oxford Univ. Press, London 1985

[4] R. T. CURTIS, A new combinatorial approach to $M_{24}$, Math. Proc. Camb. Phil. Soc. 79(1976), 25-42.

[5] M. DEHON, D. LEEMANS, Constructing coset geometries with Magma: an application to the sporadic groups $M_{12}$ and $J_{1}$, Atti Sem. Mat. Fis. Univ. Modena, to appear.

[6] The GAP Group, GAP-Groups, Algorithms, and Programming, Version 4.4:2005, (http://www.gap-system.org).

[7] N. KILIC, Residually connected geometries for $M_{22}$, Ph.D. thesis, UMIST, Manchester, 2002.

[8] N. KILIC, P. ROWLEY, On rank 2 and rank 3 residually connected geometries for $M_{22}$, Note di Matematica, 22(2003),107-154.

[9] N. KILIC, On rank 2 geometries of the Mathieu group $M_{24}$, International Journal of Pure and Applied Mathematics, to appear.

[10] N. KILIC, On rank 2 geometries of the Mathieu group $M_{23}$, Preprint, 2006.

[11] E. A. KOMISSARTSCHIK, S. V. TSARANOV, Construction of finite groups amalgams and geometries. Geometries of the group $U_{4}(2)$, Commun. Algebra 18(1990), N4, 10711117.

[12] D. LEEMANS, The rank 3 geometries of the simple Suzuki group $S z(q)$. Note Mat. 19(1999), 43-64.

[13] D. LEEMANS, The residually weakly primitive pre-geometry of the Suzuki simple groups, Note Mat. 20(2001), 1-20.

[14] D. LEEMANS, The residually weakly primitive geometries of $M_{23}$. Preprint, 2001.

[15] D. LEEMANS, The residually weakly primitive geometries of $J_{2}$. Preprint, 2001.

[16] D. LEEMANS, The residually weakly primitive geometries of $M_{22}$. Preprint, 2001.

[17] M. A. RONAN, S. D. SMITH, 2-local geometries for some sporadic groups, AMS Symposia in Pure Mathematics 37 (Finite Groups). American Math. Soc., 1980, 283-289.

[18] M. A. RONAN, G. STROTH, Minimal parabolic geometries for the sporadic groups, Europ. J. Combinatorics, 5(1984), 59-91.

[19] S. V. TSARANOV, Geometries and amalgams of $J_{1}$, Comm. Algebra 18(1990), N4, 1119-1135.

\section{Received: May 21, 2006}

